\title{
The Systemic Control of Growth
}

\author{
Laura Boulan ${ }^{1,2,3}$, Marco Milán ${ }^{4}$, and Pierre Léopold ${ }^{1,2,3}$ \\ ${ }^{1}$ University of Nice-Sophia Antipolis, 06108 Nice, France \\ ${ }^{2}$ CNRS, University of Nice-Sophia Antipolis, 06108 Nice, France \\ ${ }^{3}$ INSERM, University of Nice-Sophia Antipolis, 06108 Nice, France \\ ${ }^{4}$ 5ICREA, Parc Cientific de Barcelona, 08028 Barcelona, Spain \\ Correspondence: laura.boulan@unice.fr; leopold@unice.fr
}

Growth is a complex process that is intimately linked to the developmental program to form adults with proper size and proportions. Genetics is an important determinant of growth, as exemplified by the role of local diffusible molecules setting up organ proportions. In addition, organisms use adaptive responses allowing modulating the size of individuals according to environmental cues, for example, nutrition. Here, we describe some of the physiological principles participating in the determination of final individual size.

\section{GROWTH SPEED AND DURATION: TWO PARAMETERS THAT REGULATE FINAL BODY SIZE}

$\mathrm{D}$ uring the development of multicellular organisms, the precise control of growth is essential for ensuring the emergence of adults with correct body sizes and proper relative organ proportions. These species-specific features condition many traits of adult life, such as fitness and survival. In most vertebrates and some invertebrate species, body size cannot be modified by environmental conditions once the adult stage has been reached, meaning that adult size is fully determined by the growth that takes place during the juvenile stages. Adulthood follows a process of sexual maturation during which organisms acquire reproductive capacities. In humans, sexual maturation, or puberty, is accompanied by an intense increase in the rate of growth (growth spurt). In contrast, in holo- metabolous insects, such as Drosophila, body growth and maturation occur at distinct stages, and the beginning of maturation marks the end of the growth period. In all cases, the timing at which maturation takes place determines the final body size by constraining the length of the growth period. For example, children displaying precocious puberty are typically tall for their age because of an advanced adolescent growth spurt, but generally become shorter adults because they enter maturation and, therefore, adulthood earlier. Conversely, delayed puberty commonly leads to individuals with high stature. Similarly, artificially advancing or delaying the onset of maturation during insect development modifies the individual's final size.

During development, the activity of long-range signaling molecules-mostly hormones-is fundamental to the control of growth at the organismal scale and to the coordination of growth among developing organs. These sys-

Editors: Rebecca Heald, Iswar K. Hariharan, and David B. Wake

Additional Perspectives on Size Control in Biology: From Organelles to Organisms available at www.cshperspectives.org

Copyright (C) 2015 Cold Spring Harbor Laboratory Press; all rights reserved; doi: 10.1101/cshperspect.a019117

Cite this article as Cold Spring Harb Perspect Biol 2015;7:a019117 
L. Boulan et al.

temic factors act as relays to adjust body growth in response to environmental changes. Indeed, although overall body size is largely fixed by internal cues , the growth of developing individuals remains plastic and can adapt to external (environmental) cues, nutrition, in particular. In their target organs, long-range signals act in concert with tissue-autonomous signals to regulate three key processes that determine the final size of tissues: (1) cell growth, which increases cell mass; (2) cell proliferation, which increases cell number; and (3) cell death, which establishes the final cell number.

In conclusion, in addition to intrinsic mechanisms, which fix organ sizes and proportions for a given species, two key parameters contribute to the determination of final body size: the speed of growth (growth rate) and the duration of growth (limited by sexual maturation and the transition to adulthood). Distinct hormonal pathways regulate these two parameters: cell and tissue growth rates are controlled by hormones belonging to the family of insulin-like peptides (ILPs), whereas developmental transitions-in particular, sexual maturation-are under the control of steroid hormones. Among the many open questions that remain relative to the regulation of body size, we will focus our review on the mechanisms that allow the growth program to adapt to environmental conditions, and on those that allow coordination between growth and developmental timing. For this, we will mainly refer to recent work performed using holometabolous insects as a model.

\section{Drosophila AS A MODEL FOR STUDYING BODY GROWTH}

Insects have long served as models for the study of growth control. Two distinct approaches have been undertaken by biologists. Physiologists have been interested in understanding how hormones control the size of insects through their effect on the duration of the larval feeding period, and geneticists have explored the role of signaling pathways in controlling cell and tissue growth. Over the past 10 years, Drosophila has emerged as a model of choice to merge these two approaches in a unified effort to under- stand the basic principles of body growth and size determination (Edgar 2006; Mirth and Riddiford 2007). Drosophila's recent popularity is attributed to the successful combination of its relatively simple physiology and powerful genetics, together with a high level of conservation of the main hormones and signaling pathways controlling tissue growth and metabolic homeostasis.

During development, the feeding larva undergoes a remarkable increase in body mass that determines future adult mass and size. Larvae go through three instars before initiating maturation (metamorphosis) and the transition to adulthood. Two types of tissues develop in parallel in the larva. One group of differentiated tissues carries the physiological functions of the feeding larva and grows through successive rounds of genome duplication; this is the main driver behind the increase in body mass observed during larval stages. In parallel, a set of nondifferentiated imaginal tissues undergoes a fixed program of growth and proliferation before ultimately forming adult tissues. At the end of larval development, a major developmental transition takes place, allowing entry into metamorphosis. This is a phase of intensive tissue remodeling and differentiation, leading to the replacement of most larval tissues by adult structures.

As noted above, reaching the appropriate adult size requires two sets of mechanisms that control how fast tissues grow and when they stop growing. Although these regulations are complex and difficult to address experimentally in vertebrates, their study in Drosophila has allowed the unraveling of important principles of body growth control.

\section{CONTROLLING THE DEVELOPMENTAL CLOCK: A CENTRAL ROLE FOR THE STEROID HORMONE ECDYSONE}

The life cycle of insects is similar to that of other animal species, with four main stages: embryonic development, a juvenile growth phase, sexual maturation, and reproductive adulthood. Because each developmental transition is marked by a visible molt, insects have been 
useful models for studying the molecular mechanisms that control their timing. The steroid hormone ecdysone is the master regulator of developmental transitions in insects, and pulses of ecdysone secretion drive the progression through all of the developmental stages. Ecdysone is secreted by the prothoracic gland (PG), an endocrine tissue located within the ring gland and connected to the larval brain. The control of developmental timing by the PG relies on its capacity to integrate various environmental and developmental signals. At least two checkpoint mechanisms control the timing of metamorphosis: one is mediated by nutrient availability and the metabolic status of the larva (the so-called "critical weight"), and one depends on imaginal disc growth and maturation (the growth checkpoint) (see references for detailed discussions).

In Drosophila, the peaks of ecdysone that occur at the end of the first and second larval instars trigger molting of the larval cuticle, accommodating the increase in animal size. Subsequently, a series of low-titer hormone pulses during the L3 stage prepare the animal for metamorphosis by inducing key behavioral and developmental changes (Warren et al. 2006), including a cessation of feeding, the onset of wandering behavior, the synthesis of glue protein in the salivary glands, and the initiation of fat body autophagy (Rusten et al. 2004; Scott et al. 2004). At the end of the third instar, a high-titer ecdysone peak triggers pupariation, growth arrest, and the onset of metamorphosis (Riddiford 1993).

\section{Ecdysone Signaling}

Like vertebrate steroids, ecdysone binds to members of the nuclear receptor superfamily, which function as ligand-dependent transcription factors (King-Jones and Thummel 2005). Ecdysone acts through a heterodimer of the ecdysone receptor (EcR) and ultraspiracle (USP) nuclear receptors (Thomas et al. 1993; Yao et al. 1993). In the absence of hormone, the same heterodimer acts as a transcriptional repressor to prevent the precocious activation of metamorphic processes (Schubiger and Truman 2000;
Schubiger et al. 2005). When activated, the EcR/USP heterodimer triggers the transcription of primary (early) and secondary (late) response genes in ecdysone-target tissues. The early gene products activate the expression of the late genes but also, simultaneously, induce the expression of early-late genes that will ultimately repress these same late genes. This complex cross talk allows the sequential activation of gene expression and the progression of the signaling cascade. The termination of the ecdysone peaks relies on feedback mechanisms that decrease the circulating levels of the hormone (Rewitz et al. 2010; Guittard et al. 2011). A similar feedback mechanism in peripheral tissues also participates in the generation of the ecdysone pulse that triggers pupariation. Specific combinations of EcR isoforms and cofactors in target tissues mediate stage- and tissue-specific responses to ecdysone (Yamanaka et al. 2013). One example of such a combination is the regulation of $A D O R$, a transcriptional activator that physically interacts with EcR (Francis et al. 2010). dDOR is required for maximal EcR transcriptional activity during metamorphosis. Interestingly, $d D O R$ is a target of the transcription factor dFOXO, a negatively acting downstream component from IIS (see below), which is itself active during late larval development, when animals stop feeding. The specificity of cell and tissue responses to ecdysone is also illustrated by comparing its effects on endoreplicative (larval) and proliferative (imaginal) cells. Although EcR activation in larval tissues leads to their destruction and remodeling by autophagy and cell death (Baehrecke 2000; Lee and Baehrecke 2001; Rusten et al. 2004; Scott et al. 2004), it contributes to morphogenesis and differentiation of imaginal tissues (D'Avino and Thummel 2000; Schubiger et al. 2005).

\section{Developmental Control of Ecdysone Production}

During larval stages, the steroid hormone ecdysone is produced primarily in the PG by a series of enzymatic steps that converts cholesterol into ecdysone. The Halloween genes encode cytochrome P450 enzymes that catalyze the majority 
L. Boulan et al.

of the successive reactions of ecdysteroidogenesis (Gilbert 2004; Huang et al. 2008). Once released into the hemolymph, ecdysone is taken up by several peripheral tissues including the gut, fat body, and Malpighian tubules, where it is converted to its active form, 20-hydroxyecdysone (20E), by the P450 monooxygenase enzyme encoded by the shade gene (Petryk et al. 2003).

Multiple inputs converge on the PG to regulate ecdysone production, either coming from the central nervous system, from systemic cues relaying both intrinsic and extrinsic information or from autonomous feedback mechanisms (Fig. 1). These signals modulate ecdysone production by controlling cholesterol uptake, by regulating the expression of ecdysone biosynthetic enzymes (transcriptionally or posttranscriptionally), and by modifying the activity of other signaling pathways. Although the classic vision of ecdysone biogenesis has given a predominant role to the neuropeptide prothoracicotropic hormone (PTTH), on which most regulatory clues converge, recent results have highlighted new modes of regulation acting in parallel to PTTH on PG cells, where ecdysone is produced.

\section{PTTH}

PTTH is the primary factor that stimulates the production of ecdysone in the PG. Its production by two pairs of neurosecretory cells in the brain (Kawakami et al. 1990; McBrayer et al. 2007) is key for the generation of ecdysone pulses (McBrayer et al. 2007). Genetic ablation of PTTH cells strongly delays larval development. Surprisingly, larvae still eventually enter metamorphosis following such ablation, suggesting that PTTH is not absolutely required for metamorphosis per se, but rather that it controls final body size by regulating the timing of metamorphosis. PTTH signaling promotes ecdysone biosynthesis by promoting the expression of several Halloween genes; the expression of these genes is strongly delayed in ablated animals.

PTTH signals through the receptor tyrosine kinase Torso, which was previously identified as a master controller of terminal patterning in the embryo (Rewitz et al. 2009). Interestingly,
Trunk, the torso ligand for terminal patterning, is structurally related to PTTH, and PTTH expression in the embryo rescues terminal differentiation defects of trunk mutants. Torso/PTTH signaling relies on the activation of the canonical mitogen-activated protein kinase (MAPK)-signaling pathway, leading to the phosphorylation of extracellular signal-regulated kinase (ERK). The depletion of torso or of ERK in PG cells phenocopies the ablation of the PTTH neurons and can be rescued by feeding animals with $20 \mathrm{E}$, confirming that the delay and increased body size are indeed caused by abnormally low ecdysone levels. Consistent with this, expressing an activated form of Ras ( $\operatorname{Ras}^{\mathrm{v} 12}$ ) in PG cells activates ecdysone synthesis and changes the timing of the larva/pupa transition (Caldwell et al. 2005).

The loss of PTTH delays not only the larva/ pupa transition but also, to a lesser extent, the larva/larva molts, suggesting that PTTH plays a conserved role during all of these transitions. In addition, PTTH signaling is required for generating the small peaks of ecdysone that occur at the beginning of the third larval instar. In particular, an elegant study has shown that the nucleocytoplasmic trafficking in PG cells of the nuclear receptor DHR4, a target of the PTTH pathway, is required for these early ecdysone pulses and for the resulting changes in behavior (Ou et al. 2011). When DHR4 expression is impaired in the PG, larvae stop feeding prematurely and enter metamorphosis following a rapid increase in ecdysone levels; this suggests that the nuclear translocation of DHR4 normally blocks the occurrence of the ecdysone pulses. Further, loss of PTTH causes DHR4 to remain in the nucleus, whereas constitutive activation of PTTH signaling maintains it in the cytoplasm. Thus, PTTH acts early in the third larval instar to promote ecdysone production by preventing DHR4 from entering the nucleus of PG cells.

PTTH expression in Drosophila appears to be regulated by the photoperiod (McBrayer et al. 2007). It shows a cyclic pattern with an 8 -h periodicity, before increasing to $12 \mathrm{~h}$ before pupariation. Multiple lines of evidence suggest that the circadian clock contributes to the periodicity of ptth expression. For example, in animals mutant for $p d f$ (a gene known to regu- 


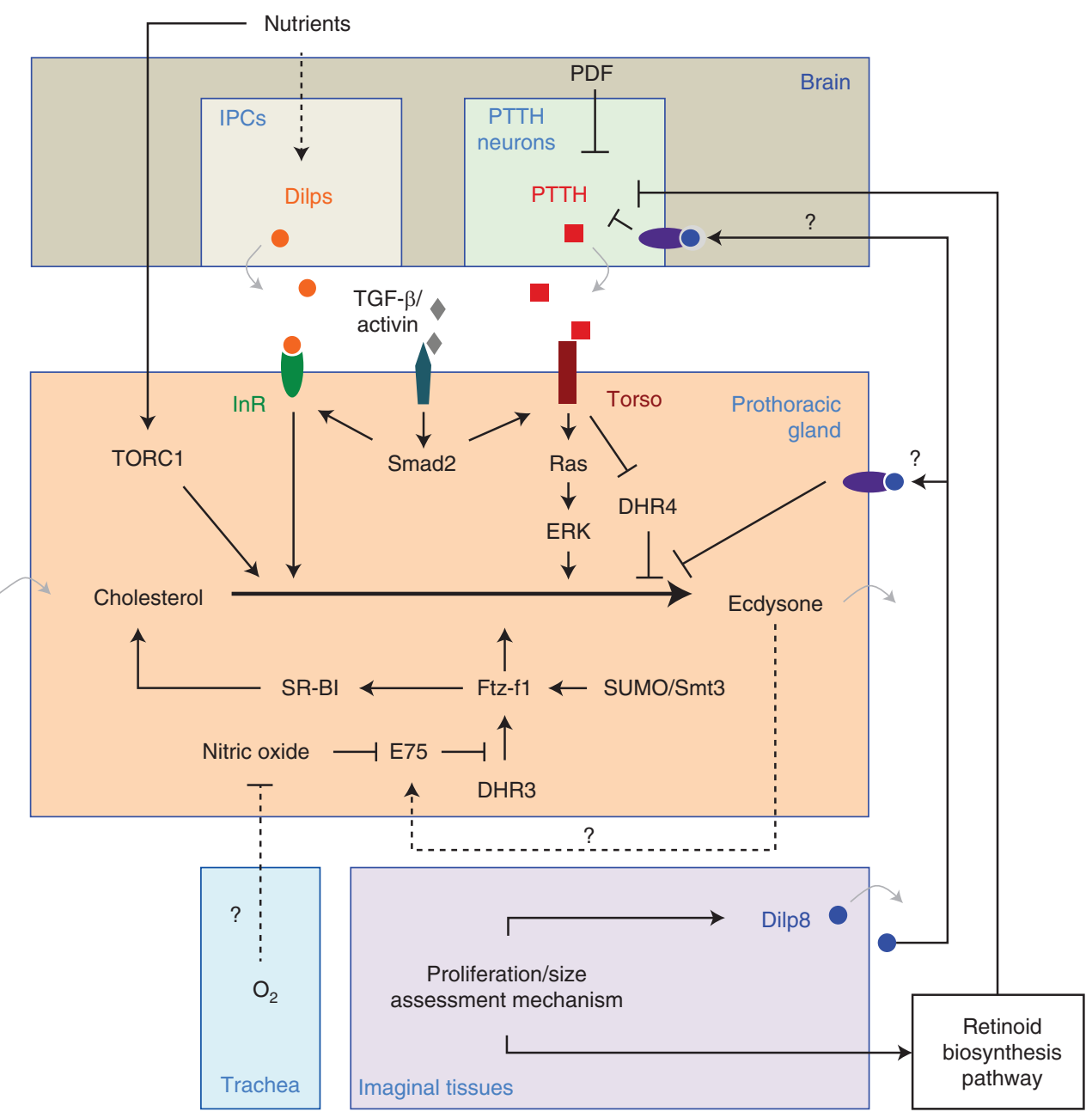

Figure 1. Environmental and developmental signals controlling steroid hormone production and the developmental timing. Developmental transitions are controlled by peaks of the steroid hormone ecdysone. Ecdysone is produced by the prothoracic gland (PG), an endocrine tissue that integrates various signals to adjust the progression through the life cycle with developmental and environmental cues. In late larval development, a sharp increase in the production of prothoracicotropic hormone (PTTH) by two pairs of brain neurons induces the production of ecdysone by the PG. Secreted factors produced by growing or regenerating tissues inhibit the larval/pupal transition, allowing coupling tissue size assessment with the developmental program. Information on body size could also come from the levels of circulating oxygen. Finally, insulin and target of rapamycin complex 1 (TORC1) signaling in the PG coordinate steroid hormone production with nutritional conditions. IPCs, insulin-producing cells; Dilp, Drosophila insulin-like peptide; InR, insulin receptor; PDF, pigment dispersing factor; DHR-3/4, Drosophila hormone receptor 3/4; ERK, extracellular signal-regulated kinase.

late circadian rhythms), PTTH oscillations are altered and $p t t h$ mRNA levels are up-regulated. Furthermore, PDF-producing neurons impinge directly on PTTH neurons. PTTH levels are also modulated by two developmental checkpoints that monitor animal size, critical weight, and the development of future adult structures (imaginal discs). The question of the developmental checkpoints controlling insect metamorphosis is addressed later in this section. It thus appears that PTTH is an important relay in the coordination of developmental 
L. Boulan et al.

timing with environmental and developmental cues, although very little is known about the mechanisms that regulate its levels.

\section{Transforming Growth Factor (TGF)- $\beta$ /Activin}

Consistent with its playing a role in promoting ecdysteroidogenesis, the activation of TGF- $\beta$ / activin signaling in the PG leads to precocious pupariation and pupal lethality (Gibbens et al. 2011), whereas inactivating this signaling pathway produces giant larvae that fail to enter metamorphosis. TGF- $\beta$ /activin has a dual function in controlling ecdysone production, as they modulate both PTTH and insulin signaling by promoting the expression of Torso and InR. Consistent with this finding, the delayed metamorphosis obtained after knocking down Smad2 in the PG can be rescued by expression of activated Ras (a downstream effector of PTTH/Torso), InR, or Akt. However, only Ras ${ }^{\mathrm{V} 12}$ expression can rescue the mRNA levels of ecdysone biosynthetic enzymes, suggesting that the two pathways act at the transcriptional and posttranscriptional stages of biosynthetic enzyme expression, respectively. Therefore, TGF- $\beta$ /activin signaling acts as a competence signal that confers high responsiveness to PTTH and insulin signals to PG cells. This regulation appears to be conserved in other model systems, because in Caenorhabditis elegans, TGF- $\beta$ /activin signaling controls the production of dafachronic acid, a functional counterpart of ecdysone (Wollam and Antebi 2011). The developmental or environmental factors that modulate TGF- $\beta$ /activin in this control are currently unknown.

\section{Nitric Oxide and E75}

Nitric oxide (NO) gas acts as a short-range signaling molecule in numerous biological processes. The nuclear receptor E75, an early puff gene in the ecdysone-signaling hierarchy, was found to contain a heme and to function as an NO sensor (Reinking et al. 2005). In target tissues, $\mathrm{NO}$ acts by inhibiting the normally repressive activity of E75A (Johnston et al. 2011). NO synthase activity is required in the PG to induce pupariation (Cáceres et al. 2011). Molecularly,
NO reverses the ability of E75 to interfere with its partner DHR3, allowing DHR3 to activate BFTZ-F1 expression, which, in turn, promotes ecdysone synthesis. Given that E75 is a target of ecdysone signaling, and that $E c R-A$ is expressed in PG cells (Talbot et al. 1993), it is likely that this NO-induced rise in ecdysone levels, in turn, negatively feeds back on the further production of ecdysone by activating E75 and, thus, downregulating $\beta F T Z-F 1$. In their study, Cáceres and colleagues point out that several cofactors required for NO synthase activity are produced on PTTH/Torso/Ras signaling. Therefore, PTTH could act through nitric oxide synthase (NOS) activation to induce the production of ecdysone. Alternatively, because NOS is activated under low oxygen (Wingrove and O'Farrell 1999), the NO pathway could serve to couple oxygen levels with ecdysone production. This would be in line with two recent reports linking oxygen levels and pupariation timing in both Manduca sexta and Drosophila models (Callier and Nijhout 2011; Callier et al. 2013). In Man$d u c a$, the size and internal volume of the tracheal network are fixed at the last larval molt. The drastic increase in larval volume and metabolic activity that takes place after this molt suggests that oxygen becomes limiting, and this could be a trigger for the pupal transition (Callier and Nijhout 2011). Consistent with this, Drosophila larvae reared in hypoxic conditions produce a peak of ecdysone at an earlier time and at a reduced mass than their normoxic controls, even though the converse condition (hyperoxic) does not lead to conclusive results (Callier et al. 2013). This, nevertheless, suggests that variations in oxygen levels could participate in the control of ecdysone production. It remains to be determined, however, whether a physiological drop in oxygen at the end of larval development constitutes a trigger for ecdysone production, and whether a NOS-dependent mechanism is involved.

\section{SUMOylation and Cholesterol Uptake}

The only Drosophila member of the SUMO family, Smt3, is required for ecdysone synthesis and, in particular, for cholesterol uptake. Down-reg- 
ulating Smt3 in PG cells lowers the lipid content of animals, prevents entry into metamorphosis, and produces giant larvae (Talamillo et al. 2008). Smt3 acts by promoting the expression of the nuclear receptor Ftz-f1, which, in turn, activates the expression of scavenger receptors (Talamillo et al. 2013). These receptors, whose functions may be conserved in the mammalian adrenal gland, mediate the regulation of lipid uptake by Smt3 in PG cells. Interestingly, Smt3 and scavenger receptors carry out similar functions in the follicle cells of female ovaries, suggesting that Smt3, Ftz-f1, and scavenger receptors may constitute a common module in the steroidogenic pathway.

Mutations in either of the two human Niemann-Pick type C (NPC) genes, NPC1 and NPC2, cause a neurodegenerative disease characterized by an abnormal accumulation of cholesterol in cells. In Drosophila, npcla and $n p c 2 a ; n p c 2 b$ mutants die during larval/pupal development because of cholesterol shortage and a lack of ecdysone (Huang et al. 2005, 2007). The expression of $n p c 1 a$ is regulated by the Broad-Complex (BR-C). Indeed, the BR-Z4 isoform directly binds to a conserved cis-regulatory module within the npcla locus, suggesting that BR-C transcriptionally controls cholesterol uptake by directly activating npcla expression (Xiang et al. 2010). Interestingly, BR-C is an early target of ecdysone, and BR-Z4 is strongly upregulated in PG cells from the wandering stage until pupariation (Zhou et al. 2004). This could constitute a positive feedback loop in the PG, in which ecdysone-mediated activation of BR-Z4 and npcla would stimulate further ecdysone biosynthesis. Overall, these forms of regulation appear to participate in a permissive control mechanism that allows the PG to benefit from the right metabolic environment before intense ecdysteroidogenesis takes place.

\section{REGULATION OF BODY GROWTH: A CONSERVED ROLE FOR THE} INSULIN/INSULIN-LIKE GROWTH FACTOR (IGF)-SIGNALING PATHWAY (IIS)

The insulin/IGF-signaling pathway (IIS) is highly conserved and regulates a number of physiological functions, such as metabolic homeostasis, growth, and longevity. In vertebrates, the metabolic functions are mediated by insulin, whereas growth is promoted by IGFs (Nakae et al. 2001). This functional division is partly explained by the differential binding of the ligands to distinct receptors (insulin receptor [IR], IGF-I receptor [IGF-IR], and IGF-II receptor), although it is also true that IR and IGF-IR receptor activation induce common signaling pathways in many cell types. Insects possess a conserved insulin-like system, which could represent a possible ancestor of the vertebrate IIS (Wu and Brown 2006). Drosophila insulin-like peptides (Dilps) carry out both the metabolic functions of mammalian insulins and the growth-promoting functions of IGFs (Garofalo 2002), and activate a conserved kinase cascade.

\section{Insulin Signaling in Drosophila}

Activation of the insulin receptor ( InR) by the Dilp ligands leads to the recruitment of Chico, the sole insulin receptor substrate (IRS)-adaptor protein in Drosophila, as well as to the activation of phosphoinositide-3-kinase (PI3K). Activated PI3K phosphorylates PIP2 (phosphatidylinositol-4,5-diphosphate) at the plasma membrane and produces PIP3 (phosphatidylinositol-triphosphate). This activity is reversed by phosphatase and tensin homolog (PTEN), a negative regulator of the pathway (Goberdhan et al. 1999). PIP3 induces the recruitment of Akt (a proto-oncogene also known as protein kinase $\mathrm{B}[\mathrm{PKB}])$ to the plasma membrane, leading to its subsequent phosphorylation. Chimeric receptors encoding the cytoplasmic domain of the Drosophila InR together with the extracellular domain of human IR bind vertebrate insulin and show tyrosine kinase activity when expressed in mammalian cells (Yamaguchi et al. 1995); this indicates that the vertebrate and invertebrate receptors share common signaling properties.

Mutations that block the activity of the pathway cause a reduction in tissue growth and lead to either lethality or severe body size reduction (Böhni et al. 1999). In the opposite direction, mutations in PTEN or overexpression 
L. Boulan et al.

of positively acting components of the pathway, such as InR, PI3K, or Akt, lead to dramatic overgrowth at both the tissue and organismal levels (Leevers et al. 1996). These results could suggest that IIS participates in the control of final body size. However, a large body of evidence indicates that IIS plays a permissive role in final size determination, coordinating the intrinsic growth program with nutritional conditions.

\section{The Dilps}

Eight ILPs have been identified to date in Drosophila. With the exception of the recently identified Dilp8 (Colombani et al. 2012; Garelli et al. 2012), all of the other Dilps activate the canonical fly IIS pathway and promote cell growth and anabolism. Overexpression of any of the Dilps 1-7 leads to increased larval size in an InR-dependent manner (Ikeya et al. 2002). Conversely, homozygous mutant animals presenting a deletion of the main dilp gene cluster (dilp1-5) show growth and metabolic defects (Zhang et al. 2009; Grönke et al. 2010) pointing to a functional involvement of Dilps in the regulation of growth and metabolic homeostasis.

\section{Temporal and Spatial Expression of Dilps}

Quantitative reverse transcription polymerase chain reaction (RT-PCR) analysis on whole animals has revealed that members of the dilp gene family display different temporal expression patterns. Although dilp1, 3 and 6 are expressed at high levels during early pupal stages, dilp2 and 5 display high levels during the larval growth period (Okamoto et al. 2009; Slaidina et al. 2009). The dilps are also expressed in various larval tissues. For example, dilp1, 2, 3, and 5 transcripts are found in two symmetric clusters of seven neurosecretory cells in the larval brain (hereafter referred to as the insulin-producing cells [IPCs]), which are functionally homologous to the vertebrate $\beta$ cells (Brogiolo et al. 2001; Ikeya et al. 2002; Rulifson et al. 2002). Lineage analysis of these cells in the developing central nervous system has shown that precursor neuroblasts of both the insulin-producing cells and the glucagon (AKH in flies)-producing cells express a transcription-factor cascade that is orthologous to that expressed in the adenohypophyseal placode of vertebrates. Therefore, a rudimentary endocrine axis might have evolved from a common ancestor of flies and vertebrates to control insulin and glucagon-like functions (Wang et al. 2007).

Although located in the same gene cluster, dilp2, 3, and 5 present independent regulated expression in the IPCs (Ikeya et al. 2002). Whereas dilp3 and dilp5 transcripts decrease on starvation, dilp2 transcription is insensitive to nutrient levels (Ikeya et al. 2002; Colombani et al.2003). dilp5 is also expressed in the midgut, dilp2 in the imaginal discs and the salivary glands, dilp 6 in the fat body, and dilp7 in the ventral nerve cord (Brogiolo et al. 2001). Thus, although the different dilp genes can replace each other functionally, their specific expression patterns suggest that they carry out distinct physiological functions in vivo.

\section{Local Signaling in the Central Nervous System}

In addition to the systemic role that ILPs play, recent studies have highlighted other local, short-range functions for several of them (Fig. 2) (Cheetham and Brand 2013). At the end of embryonic development, neural stem cells enter a period of quiescence that precedes their active proliferation during the larval stages. Dilp2 and Dilp6 are found within a population of glial cells and are required to stimulate neural stem cell proliferation after the first larval feeding (Chell and Brand 2010). The activation of dilp gene expression in glial cells indirectly depends on dietary amino acids. This regulated expression is mediated by a target of rapamycin (TOR)-dependent-sensing mechanism that takes place in fat body cells and is relayed to the brain glia by an unknown humoral signal (Sousa-Nunes et al. 2011). A recent study has revealed that gap junctions between the glial cells that form the bloodbrain barrier coordinate nutrient-dependent calcium oscillations, which are required for the local production and secretion of ILPs (Spéder and Brand 2014). Interestingly, this local release of Dilps by glial cells is incapable of regulating systemic larval body growth, and IPC-derived 


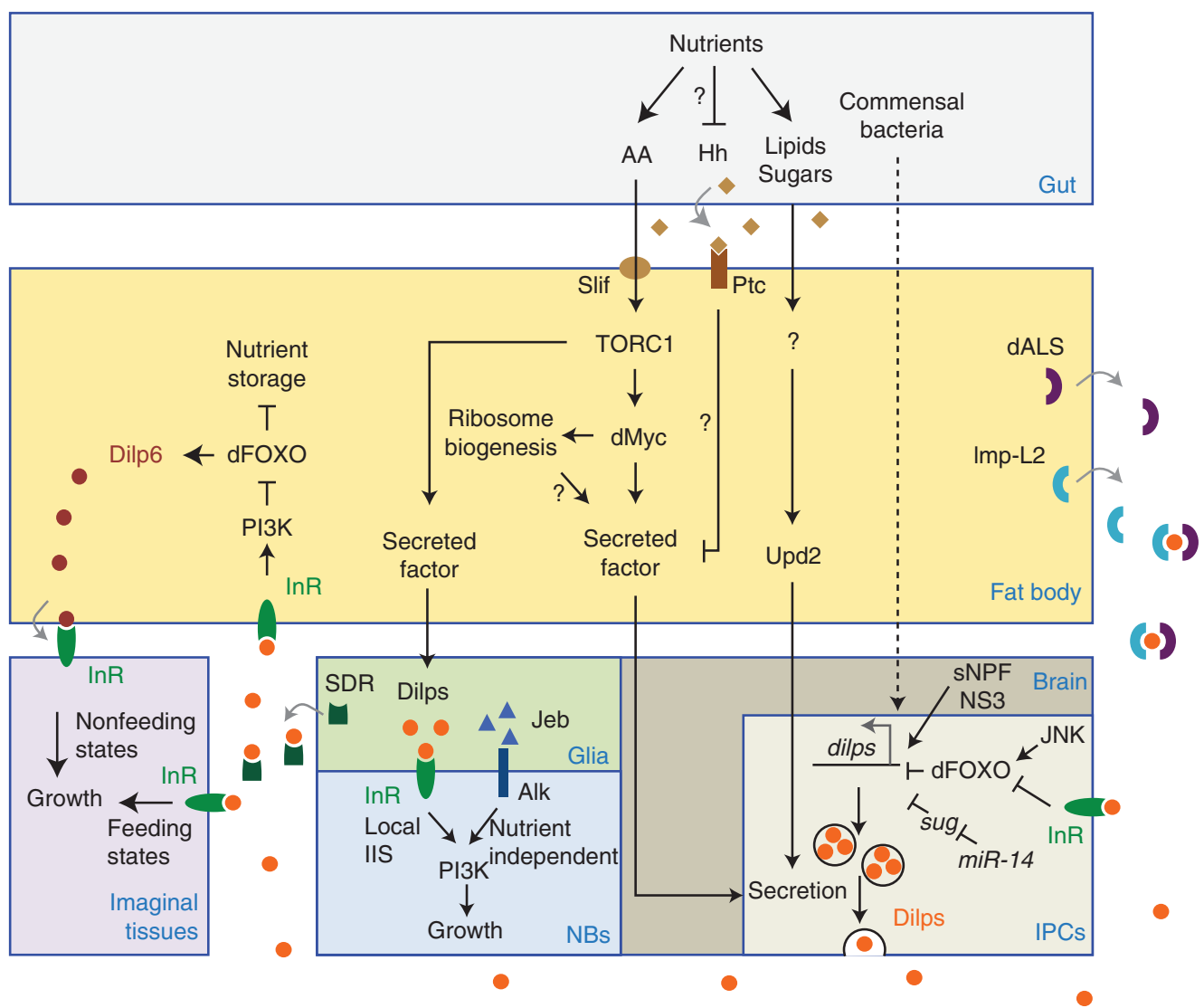

Figure 2. Local and systemic modulations of insulin signaling. The tight control of insulin signaling relies on complex mechanisms of interorgan communication. The insulin-producing cells (IPCs) produce Drosophila insulin-like peptide (Dilp)2,3, and 5, which represent the main systemic source of circulating insulin during the growth phases (larval stages). Several inputs affect dilp gene expression in the IPCs, whereas nutritional information is sensed by the fat body and relayed by humoral factors controlling Dilp secretion. Dilp6 is induced upon nutrient shortage by fat cells to sustain growth of high priority organs. Several Dilp-binding factors modulate the activity of circulating Dilps (SDR, dALS, and Imp-L2). An interplay between glial and neural cells promotes growth and proliferation of larval neuroblasts and allows them to evade nutrient control during late larval development. NBs, neuroblasts; PG, prothoracic gland; AAs, amino acids; Hh, Hedgehog; FOXO, Forkhead box-O transcription factor; PI3K, phosphoinositide-3-kinase; Upd2, unpaired 2; Imp-L2, imaginal morphogenesis protein-Late 2; dALS, Drosophila acid labile subunit; Alk, anaplastic lymphoma kinase; SDR, secreted decoy of InR; Jeb, Jelly belly.

Dilps in the hemolymph do not induce the reactivation of neuroblasts. Therefore, local and systemic Dilps are used to trigger separate outputs in response to nutritional status.

\section{Functional Characterization of Dilps}

The dilp6 gene is expressed at high levels in the fat body during the late larval stage (wandering period) and the pupal period (Okamoto et al. 2009; Slaidina et al. 2009). Functional analysis reveals that dilp6 is induced under conditions of nutrient deprivation, caused either by developmentally induced cessation of feeding or by starvation, to promote tissue growth. Accordingly, dilp6 mutants hatch as smaller adults and present a greater size reduction after starvation than do wild-type animals. Adipose expression 
L. Boulan et al.

of dilp6 under starvation relies on Drosophila forkhead box-O transcription factor (dFOXO), which accumulates in an active, nuclear form upon nutrient deprivation. Induction of dilp6 at the larval/pupal transition is independent of $\mathrm{dFOXO}$ and is controlled rather by ecdysone peaking before metamorphosis. On the basis of these studies and according to structural features that distinguish it from other fly insulins, Dilp6 was proposed to be an analog of vertebrate IGF-I. Indeed, IGF-I is present at high levels during puberty and is regulated by nutrition and sex steroids.

dilp2, 3, and 5 are expressed at high levels in the brain IPCs during larval stages. Ablation experiments indicate that larval IPCs are the main supply for systemic insulins and are essential for maintaining growth and carbohydrate homeostasis (Rulifson et al. 2002). Interestingly, the reduction in larval growth and adult size observed upon IPC ablation can be rescued by ubiquitous expression of dilp2 alone, suggesting that brain-derived Dilps may be partially redundant (Rulifson et al. 2002). In line with this result, no defects in final body size are observed upon Dilp2 knockdown in the IPCs (Broughton et al. 2008). A deeper analysis of dilp gene function, made possible by the generation of knockout mutations (Grönke et al. 2010), has revealed complex compensatory regulations acting at the transcriptional level: dilp5 is up-regulated in dilp2 and dilp2-3 mutants, and dilp3 is up-regulated in dilp2 and dilp5 mutants. Individual knockouts of brain dilps do not affect general insulin signaling, nor do they induce organismal growth defects. However, overall insulin signaling and body size are severely reduced in dilp2, 3, and 5 mutants, consistent with the data from IPC ablation. Strikingly, dilp6 expression is strongly induced in dilp2-3, 5 mutants, suggesting that this condition could mimic a starvation-like state.

\section{Control of Dilp Production in the IPCs}

In mammals, insulin levels are modulated by hormones and neurotransmitters acting on its release from $\beta$ cells (Drucker et al. 1987; Aspinwall et al. 1999; Adeghate et al. 2001; Dong et al.
2006; Sonoda et al. 2008). Similarly, in flies the production and release of Dilps by the brain IPCs seems to be controlled by both nutritiondependent and independent cues (Fig. 2). We will detail the nutrition-dependent control of Dilp function below. In addition, the Drosophila IPCs receive many inputs from neurons producing neuropeptides or neuromediators, the function of some of which is possibly linked to the processing of nutritional information.

Drosophila short neuropeptide F (sNPF), a homolog of mammalian neuropeptide $\mathrm{Y}$ (NPY), controls food intake. Recent work has indicated that this peptide promotes body growth by regulating dilp expression (Lee et al. 2008). sNPF is expressed in adjacent neurons and signals to the IPCs through the sNPF receptor 1 , inducing expression of dilp genes. This suggests a possible feedback control mechanism linking feeding behavior to insulin signaling, although the physiological context of such feedback has not yet been clarified. Such a mechanism would also parallel the case of rat insulinoma cells, in which NPY signaling promotes the expression of insulin in an ERK-dependent manner, suggesting a conserved regulatory mechanism.

Another neuronal mechanism regulating IPC activity involves serotonergic circuits. NS3, a Drosophila nucleostemin family GTPase (Kaplan et al. 2008), is required in serotonergic neurons - some of which are closely apposed through their axonal projections to the IPCsto regulate final body size. Mutant ns3 animals present a reduced size and an accumulation of Dilp2 in the IPCs, suggestive of a defect in Dilp release. Expression of $n s 3$ in these 5-HT neurons is sufficient to rescue the growth defects and the developmental delay observed in $n s 3$ mutant animals. However, it is not clear yet whether the lack of NS3 function leads to an increase or a decrease in serotonin signaling, what is the precise identity of the 5-HT neurons that control IPC function, and under what physiological conditions serotonin is used to control Dilp release. In line with these results, the 5HT1A receptor is expressed in the IPCs, in which its specific knockout leads to a variety of phenotypes reminiscent of both increase 
and decrease in insulin signaling (Luo et al. 2012, 2014).

The knockdown of specific receptor-encoding genes in the IPCs has revealed that other neuromodulators like GABA, tachykinin, and octopamine can also modulate IPC function (Crocker et al. 2010; Enell et al. 2010; Birse et al. 2011). After this initial phase defining the possible signaling pathways at play, the next challenge will obviously be to put these different mechanisms into their physiological context.

Drosophila miR-14 is expressed in the IPCs of adult flies, in which it promotes the expression of dilp2, 3, and 5 and controls metabolism (Varghese et al. 2010). The combination of expression profiling and target prediction analysis has led to the identification of sugarbabe as the functional target of miR-14. Interestingly, sugarbabe is down-regulated in starved animals independently of miR-14, meaning that its expression in the IPCs is regulated by two independent means. Overexpressing sugarbabe enhances the decrease in dilps transcript levels observed upon starvation, indicating that its down-regulation in this condition acts to limit the negative effects of nutrient deprivation on dilps expression.

Finally, insulin signaling in the IPCs positively regulates the secretion of systemic Dilps, linking the levels of activation of the pathway to the production of the ligand (Cheng et al. 2011). This data contrasts with the observation that, in adult flies, Dilp6 produced by fat cells increases longevity and longevity-associated metabolic phenotypes by repressing dilp2 and dilp5 expression in the brain IPCs (Bai et al. 2012). This suggests that in this latter case, Dilp6 might not act directly on the brain IPCs.

\section{Nutritional Control of Body Size}

Nutrition is a key environmental parameter in the determination of body size. In multicellular organisms, nutritional information is conveyed at both the cellular and the systemic levels to achieve balanced growth. The coordination of growth among different body parts according to environmental conditions relies on cross talk among different organs or tissues through the production of long-range signaling molecules
(Mirth and Shingleton 2012; Andersen et al 2013). Among these organs, the fat body plays a key role in orchestrating organism-wide responses to nutritional status (Fig. 2). In addition to its function in accumulating energy resources that will be used during metamorphosis (by storing sugars in the form of glycogen and fat in the form of triacylglycerol [TAG]), the fat body integrates and emits signals involved in the regulation of growth and metabolism. The insulin/IGF and TOR pathways are the main players involved in sensing and relaying nutritional information to coordinate growth with nutrient availability. Information about nutritional status is sensed directly at the level of individual cells via the TOR pathway, whereas the systemic response is performed by the hormonal action of insulin.

\section{The TOR-Signaling Pathway Acts as a} Conserved Cell-Based Nutrient Sensor

The role of the protein kinase TOR in mediating cellular nutrient sensing is conserved from yeasts to mammals (De Virgilio and Loewith 2006). Both cell size and cell proliferation are regulated by TOR signaling (Zhang et al. 2000). TOR activity is controlled by the small GTPase Rheb and Rheb mutant flies display severe undergrowth as a consequence of reduced cell size and cell number (Saucedo et al. 2003; Stocker et al. 2003). The tumor suppressors tuberous sclerosis complex genes 1 (TSC-1) and 2 (TSC-2) act together in a complex to inhibit Rheb activity (Inoki et al. 2003a; Tee et al. 2003; Zhang et al. 2003). In Drosophila, clones of cells mutant for TSC-1/2 display strong overgrowth (Potter et al. 2001; Tapon et al. 2001), consistent with increased Rheb and TOR activities. The TOR kinase is found in two complexes, TORC1 and TORC2 (Loewith et al. 2002). TORC1 is the primary mediator of energy and amino-acid sensing and couples growth cues to cellular metabolism (Wullschleger et al. 2006). TORC1 promotes tissue growth by adjusting protein biosynthesis, through the regulation of the two translational regulators S6K (ribosomal protein kinase 6) and 4E-BP (initiation factor $4 \mathrm{E}$ binding protein). TOR-mediated inhibition 
L. Boulan et al.

of $4 \mathrm{E}-\mathrm{BP}$ and activation of S6K enable the assembly of the translation preinitiation complex and stimulate peptide chain elongation, thereby enhancing protein synthesis (Wang et al. 2001; Holz et al. 2005). Inhibiting TORC1 function in Drosophila reduces body growth and causes larval lethality (Zhang et al. 2000). However, flies lacking S6K or 4E-BP activities alone display significantly milder phenotypes (Miron et al. 2001; Teleman et al. 2005), indicating that TORC1 has additional functions in vivo. Indeed, TORC1 also regulates transcription, mainly through the posttranscriptional activation of the dMyc transcription factor (Teleman et al. 2008). Consistent with a role for dMyc in promoting ribosome biogenesis (Grewal et al. 2005), the majority of TORC1regulated genes are involved in the production of ribosomes and modulate cell growth (Guertin et al. 2006). This dual activity of TORC1 allows the rate of translation to be coupled with the amount of ribosomes.

TORC1 is modulated by cellular aminoacid levels, thereby linking nutritional status with the rate of protein synthesis (Avruch et al. 2009). The amino-acid-dependent activation of TORC1 takes place downstream of the TSC- $1 / 2$ complex and is mediated by Rag GTPase proteins. The Rag complex is required for the translocation of TORC1 to the lysosomal surface, which is a crucial step for TOR activation (Kim et al. 2008; Sancak et al. 2008, 2010). Under amino-acid deprivation, activation of mTORC1 is abolished by a negative regulator of the pathway, SH3BP4, which inhibits the formation of active Rag GTPase complexes (Kim et al. 2012). The adaptor protein p62 is also required to mediate the interaction between mTORC1 and the Rag complex in an aminoacid-dependent manner (Duran et al. 2011). Another study in Drosophila has proposed that MAP4K3 also participates in the regulation of Rag complex activity by amino acids (Bryk et al. 2010).

TORC1-mediated cell growth is also under the control of an energy-sensing mechanism that depends on AMPK (AMP-activated protein kinase). AMPK activity monitors the immediately available energy by measuring cellular ATP levels (Hardie 2007) as well as reserve energy stores in the form of glycogen (McBride et al. 2009). AMPK signaling regulates growth by phosphorylating and activating TSC-2, which results in TORC1 inhibition (Inoki et al. 2003b).

\section{Links between Insulin and TOR Pathways}

The cross talk between IIS and TORC1 constitutes a connection between the systemic and the cell-intrinsic nutrient-sensing modules. However, this connection has been a matter of debate. In human and Drosophila cultured cells, treatment with insulin leads to rapid TORC1 activation as a result of the direct phosphorylation of TSC-1/2 and inhibition by Akt (Inoki et al. 2002; Potter et al. 2002). At the same time, in vivo studies in Drosophila have challenged the relevance of this link under physiological conditions. Indeed, the removal of all Akt phosphorylation sites from TSC- 1 and TSC- 2 does not lead to an increase in TORC1 activity, as judged by an absence of a growth increase in vivo (Dong and Pan 2004; Schleich and Teleman 2009); this result indicates that, under physiological conditions, Akt function does not contribute to TORC1 activation. More recently, the protein PRAS40 (proline-rich Akt substrate 40) was proposed to link IIS to TORC1 in cultured cells (Sancak et al. 2007; Vander Haar et al. 2007). PRAS40 binds to TORC1 and prevents it from interacting with its substrates. Phosphorylation by Akt on a single site relieves this inhibition and is necessary for the insulin-induced activation of TORC1. The identification of the Drosophila homolog of PRAS40 has allowed the generation of mutant animals and the study of its involvement in the regulation of TOR signaling in vivo (Pallares-Cartes et al. 2012). Consistent with a role as an inhibitor of TORC1, overexpression of PRAS40 reduces tissue growth in an autonomous manner by reducing cell size. However, PRAS40 null mutant flies are viable and present no obvious alterations of cell or body growth, suggesting that PRAS40 does not regulate TORC1 activity during the growth period in Drosophila. Further analysis of adult PRAS40 ${ }^{-}$mutant females revealed that PRAS40 represses TORC1 in the ovaries but not in the adult soma. In line with 
this, the ubiquitous reduction of insulin signaling decreases TORC1 activity in a PRAS40-dependent manner only in the ovaries, and removing PRAS40 in chico mutants rescues female fertility but not small body size. In conclusion, this in vivo study in flies shows that IIS and TORC1 are linked through PRAS40 specifically in nonsomatic issues.

Although the direct interplay between TOR and the insulin pathway has been challenged in the context of autonomous cell growth control, it is clear that functional interactions between these pathways play key roles in the regulation of systemic growth.

\section{The Fat Body as the Main Nutrient-Sensing Organ}

In both flies and mammals, nutrient deprivation leads to growth retardation as a consequence of reduced IIS signaling. It has been proposed that specific organs, called "sentinel tissues" (Arsham and Neufeld 2006), could act as specific nutrient sensors that would systemically modulate the levels of insulin/IGF signaling and thereby adjust body growth in response to nutrient availability. A genetic screen in Drosophila for growth-related genes identified slimfast (slif), a genetic locus encoding a conserved amino-acid transporter that mediates arginine and lysine uptake in various tissues (Colombani et al. 2003). Chronic depletion of Slif specifically in the fat body mimics the effects of amino-acid starvation, provoking general growth defects and lethality. A mild down-regulation of slif function allows the eclosion of adults with reduced size and body weight but with normal body proportions, indicating that the fat body acts as a nutrient-sensing tissue that coordinates the harmonious growth of all body parts. The sensor mechanism in fat cells relies on TORC1, because depleting fat cells of amino acids leads to a reduction in adipose TORC1 activity, and reducing TORC1 function specifically in this tissue mimics the systemic growth defects observed on slif knockdown. This study also suggested the existence of humoral signals produced by fat cells that systemically control organ growth (see below). A large part of the response to TOR signaling in the fat body is mediated by dMyc (Teleman et al. 2008), a transcription factor that promotes protein synthesis via the regulation of ribosome biogenesis (Grewal et al. 2005) and which was found to be a common target of TOR and insulin signaling (Teleman et al. 2008). The control of systemic growth by adipose TOR signaling also involves the inhibition of the repressor Mafl, leading to the activation of RNA polymerase III and tRNA synthesis (Marshall et al. 2012; Rideout et al. 2012), as well as the expression of dref, a gene encoding a transcription factor that controls the expression of ribosome biogenesis genes (Killip and Grewal 2012).

\section{Remote Control of Dilps Secretion}

The first humoral relay connecting the fat body to peripheral tissues involves the Dilps themselves (Fig. 2). During larval development, diet modulates the circulating levels of brain Dilps (Géminard et al. 2009). Upon full starvation, Dilp2 and Dilp5 are retained and accumulate in the IPCs; subsequently, refeeding larvae with amino acids only, but not sugar or lipids, releases the secretion block. Similarly, silencing slif or inhibiting TOR signaling specifically in the fat cells induces Dilp retention. In the other direction, activating TOR signaling in the fat body of underfed animals is sufficient to stimulate Dilp2 secretion. Therefore, TOR signaling in the fat body integrates nutritional inputs and controls the release of Dilps from the IPCs. Ex vivo coculture experiments further showed that the fat body secretes one or more humoral factors that remotely control insulin secretion from the brain IPCs depending on the nutritional state (Géminard et al. 2009).

The cytokine unpaired $2(\mathrm{Upd} 2)$ has recently been identified as a fat body-derived factor that promotes insulin secretion from the IPCs in both larvae and adults (Rajan and Perrimon 2012). In fed animals, Upd2 produced by fat cells navigates through the hemolymph and activates JAK/STAT signaling in a few GABAergic neurons located in the brain in close proximity to the IPCs. JAK/STAT activation in these neurons relieves the GABA-dependent tonic inhibi- 
L. Boulan et al.

tion of the IPCs and, as a consequence, stimulates Dilp secretion. In adult flies experiencing prolonged starvation, adipose $u p d 2$ expression is strongly reduced, leading to Dilp retention and decreased levels of stored TAG (lipid storage). In contrast, acute starvation does not inhibit upd2 expression, indicating that the Upd/ JAK/STAT pathway participates in a metabolic adaptation to a prolonged starved state. Upd2 presents structural similarities to leptin, a mammalian cytokine produced by adipose cells under conditions of nutritional surplus. Interestingly, Rajan and Perrimon report that expressing human leptin in Drosophila fat cells rescues the lipid metabolism defects observed in upd 2 mutant flies. This provides evidence for an evolutionarily conserved role for adipose cytokines in orchestrating metabolic responses to nutrient availability. Fat $\mathrm{Upd} 2$ is produced in response to dietary lipids and sugar, but does not respond to amino acids (Rajan and Perrimon 2012). Thus, additional humoral signals produced by fat cells presumably act to regulate Dilp secretion in response to amino acids and TOR signaling. Taken together, these studies establish a model whereby the global nutritional state is relayed by multiple factors originating in the fat body to modulate metabolic homeostasis in part by controlling Dilp production and release.

\section{Larval versus Adult IPCs}

Even though some of the studies described here indiscriminately used adults or larvae for their analyses, functional differences exist between the insulin-producing cells at these two stages, pointing to fundamental differences in their respective physiologies. As an example, the larval IPCs do not express two major components of the glucose response, namely, the Kir6 and Sur1 subunits of the $\mathrm{K}_{\text {ATP }}$ channels (Kim and Rulifson 2004); not surprisingly, these cells do not directly respond to carbohydrates in ex vivocultured brains (Géminard et al. 2009). This contrasts with adult IPCs, in which it has been reported that Sur is expressed (Fridell et al. 2009), that glucose is responsible for direct excitability (Kréneisz et al. 2010), and that Glut1 function is required for Dilp secretion (Park et al. 2014). Therefore, in contrast to larval IPCs, adult IPCs seem to share some fundamental glucose response properties with pancreatic $\beta$ cells. Although a link between adult IPC excitability and Dilp secretion has not yet been established, this finding suggests that changes in IPC function precede major physiological differences between the larval and adult stages. Specifically, during the larval stage, when organismal growth takes place, larval IPCs mainly provide Dilps to couple tissue growth with nutrient availability. In this context, carbohydrate and lipid homeostasis are maintained by a glucagon-like hormone called $\mathrm{AKH}$ that is produced in the corpus cardiacum, where cells express bona fide Sur and Kir and sense glucose (Kim and Rulifson 2004). In contrast, adults do not grow, and therefore no longer use Dilps to promote tissue growth. Accordingly, adult IPCs undergo a functional switch that allows metabolic homeostasis to be controlled in part through the expression of a set of specialized enzymes that directly sense nutrients.

In addition to intracellular sensors directly linking insulin production to the availability of nutrients, insulin secretion by mammalian $\beta$ cells is regulated by enteroendocrine hormones, called incretins, and by paracrine signals like somatostatin from adjacent islet $\delta$ cells. In adult flies, the corpora cardiaca is comprised of enteroendocrine cells that secrete $\mathrm{AKH}$ in response to fasting, and that directly contact IPCs. In addition, studies by the Kim group now show that the adult corpora cardiaca also produces peptides that regulate insulin production and secretion by IPCs during fasting and refeeding (Alfa et al. 2015). Thus, flies may use hormonal signaling with features resembling the enteroinsular axis in mammals to control insulin output in dynamic nutrient states. Whether this freshly added mechanism also participates in systemic growth control through Dilp secretion during larval development is currently unknown.

\section{Modulating the Activity of Circulating Dilps}

In vertebrates, IGF-1 accumulates in body fluids and associates with molecules referred to as 
IGF-binding partners (IGFBPs). These factors play important roles in the control of the biological activity of IGF-1 (Duan and Xu 2005). Drosophila Imp-L2 shows sequence homology with mammalian IGFBP-7 and was the first insulin-binding protein characterized in invertebrates (Honegger et al. 2008). Imp-L2 is a secreted protein that inhibits growth nonautonomously. It acts by binding directly to Dilp2 and inhibiting its activity. Accordingly, ubiquitous overexpression of Imp-L2 reduces adult size, whereas Imp-L2 mutant animals are bigger than normal. Under starvation conditions, Imp-L2 expression is induced in the fat body and is required to adjust systemic levels of insulin signaling. Imp-L2 expression is down-regulated in dilp2-3, 5 mutants (Grönke et al. 2010), suggesting the existence of a feedback mechanism between the Dilps and their repressor. Dilp2 and Imp-L2 are found in a ternary complex with the fly ortholog of acid-labile subunit (ALS), a scaffold molecule involved in the stabilization of the IGF/IGF-BP complexes in mammals (Domené et al. 2009). As in mammals, the recruitment of dALS to the complex in Drosophila requires the prior formation of a dimeric Dilp/Imp-L2 complex, indicating that Imp-L2 serves as a molecular bridge between dALS and Dilp2. dALS is produced in the fat body and is secreted into the hemolymph, where it antagonizes Dilp function for the control of growth and metabolism (Arquier et al. 2008). Surprisingly, when nutrients are limiting, increased $d A L S$ expression rescues the larval growth defect, whereas $d A L S$ silencing enhances it (Arquier et al. 2008). Therefore, although dALS antagonizes Dilps function in well-fed animals, it is limiting for proper insulin signaling under conditions of nutritional stress. Such a dual effect can be interpreted in light of the complex functions that ALS has in mammals, as it both stabilizes circulating IGF-I complexes and inhibits IGF-I function by preventing binding to its receptor.

An intriguing extension of the role of ImpL2 in regulating IIS recently came up from the independent work of two groups demonstrating that this IGF-BP is produced at high level by fly tumors to induce wasting of nontumorous tis- sues. Indeed, although organ growth and homeostasis are influenced by systemic signals under normal physiological conditions, abnormal tissue growth can alter these signals, leading to pathological conditions and eventual death. Bilder and colleagues show that malignant fly tumors transplanted into adult Drosophila induce a dramatic loss of adipose and gonadal mass, along with compromised muscle function, before the death of the host. The investigators identify Imp-L2 as a tumor-produced secreted factor that drives wasting by interfering with insulin signaling (Figueroa-Clarevega and Bilder 2015). Perrimon and colleagues also report that localized overproliferation induced by activation of Yorkie, the Yap1 oncogene ortho$\log$, in adult intestinal stem cells, causes wasting of ovary, fat body, and muscle. This wasting phenotype is associated with reduction of systemic insulin/IGF signaling via secreted Imp-L2, which is specifically elevated in the overproliferating gut. Expression of the glycolytic enzymes and the insulin/IGF pathway components are up-regulated in the overproliferating gut, suggesting a mechanism for this tissue to evade the action of Imp-L2 (Kwon and Perrimon 2015). Interestingly, these wasting phenotypes have resemblances to human cancer cachexia and may provide a simple invertebrate model for aspects of this devastating and difficult-totreat condition.

An additional, new type of Dilp-interacting molecule has recently been identified that shows a different strategy for controlling Dilp function (Okamoto et al. 2013). The secreted decoy of insulin receptor (secreted decoy of InR [SDR]) is structurally similar to the extracellular domain of the Drosophila insulin receptor (InR), and binds to circulating Dilps in the hemolymph. Experimental data reveal that SDR serves as a sink, preventing Dilps from interacting with their cognate receptor and inhibiting general insulin signaling. Interestingly, SDR and Imp-L2 compete for binding to Dilp2, suggesting that they form independent complexes with Dilp2. SDR is produced by glial cells and the knockdown of SDR in the glia is sufficient to increase animal size, whereas constitutive overexpression reduces it. Intriguingly, SDR expres- 
L. Boulan et al.

sion is independent of the nutritional status of the larvae, raising the question of what is the physiological context of its action.

\section{Other Nutrient-Sensing Organs}

The gut is directly exposed to the ingested diet and is, therefore, expected to play important functions in sensing and transducing nutritional information (Fig. 2). Indeed, pioneering work on the "incretin effect" in mammals has revealed that glucose is more potent at triggering insulin release when ingested than when injected into the blood (McIntyre et al. 1964). The last decade has seen the identification of a number of epithelial receptors and transporters similar to the ones found in the external sensing organs (tongue, nose) and able to transduce signals controlling general metabolism and feeding in response to fat, amino acids, and carbohydrates (Miguel-Aliaga 2012). Despite the high level of both anatomical and functional conservation between the vertebrate and invertebrate intestinal systems, the identification of these receptors and transporters and the genetic analysis of their function in the invertebrate gut await experimental testing. Recent work by the Eaton group has shed new light on the role of Hedgehog $(\mathrm{Hh})$, a molecule with recognized functions in tissue morphogenesis, as a metabolic hormone produced by the gut. Hh produced by midgut enterocytes can be found in the hemolymph attached to lipoproteins. Its production increases in response to nutritional deprivation, and circulating $\mathrm{Hh}$ is required for normal survival under starvation conditions. Circulating $\mathrm{Hh}$ signals to the fat body to decrease larval growth and promote TAG mobilization. It also signals to the PG to inhibit ecdysone production and delay pupariation (Rodenfels et al. 2014). These provocating findings implicate for the first time in the fly model the gut as a nutrient-sensing organ directly involved in adapting metabolism to the nutritional environment. Future work should decipher the mechanism of nutrient sensing taking place in gut cells leading to Hh production, as well as the role of $\mathrm{Hh}$ signaling in endocrine tissues relaying the nutritional input.
During recent years, skeletal muscle has also been recognized as a secretory organ, responding to changes in metabolic status by expressing myokines that communicate with and modify the status of other important metabolic tissues, like adipose, liver, $\beta$ cells, or epithelia (Pedersen and Febbraio 2012). In flies, genetic interventions on skeletal muscles modify organismal growth and aging. Muscle-targeted inhibition of IIS, for instance, reduces muscle fiber size, but also organismal size (Demontis and Perrimon 2009). The possible myokines involved in this systemic control are not known. Recent work by the Perrimon group has identified myoglianin, a myokine with homology to human GDF11 and myostatin that remotely controls rRNA levels and the size of the nucleolus in other tissues. In adults, increased expression of myoglianin in squeletal muscles leads to p38 MAPK activation, reduced nucleolar function in fat body cells and a general increase in lifespan, whereas reduction of myoglianin expression has opposite effects (Demontis et al. 2014). Whether this myokine could participate in systemic growth control during larval stages is currently unknown.

The brain is also an important sensor of nutrients and the main site for the so-called "homeostatic control of food intake," matching the quality and quantity of the food sources with organismal levels. In mice and flies, specific brain neurons evaluate various types of nutrients and energy balance through the use of sensory receptors, and control feeding behavior according to this nutritional information. So far, two types of internal sensors have been described in the fly brain. The gustatory receptor Gr43a senses both dietary fructose through taste neurons and circulating fructose in the hemolymph through a population of neurons present in the adult brain (Miyamoto et al. 2012). By sensing fluctuating fructose levels, the brain neurons promote feeding in hungry flies and suppress feeding in satiated ones. Besides carbohydrates, adult flies also sense amino acids and a deprivation of amino acids induces a change in their feeding preference (Ribeiro and Dickson 2010; Vargas et al. 2010; Toshima and Tanimura 2012). In larval brain, a specialized 
circuitry consisting of three dopaminergic neurons senses hemolymph content in essential amino acids. Upon feeding on an imbalanced diet lacking essential amino acids, this dopaminergic circuitry is activated by a GCN2-dependent molecular mechanism. This activation, in turn, triggers feeding arrest, therefore allowing animals to seek a better food source (Bjordal et al. 2014).

Finally, recent work reveals a major role for the symbiotic gut flora in controlling organismal growth. In poor nutrient conditions, commensal flora becomes limiting and its depletion causes further reduction in larval growth rate through TORC1 inhibition in the fat body (Shin et al. 2011; Storelli et al. 2011). A reduction of ecdysone production by the PG is also observed, thereby affecting the duration of larval growth (Storelli et al. 2011). The nature of the signal linking the activity of the gut flora and TORC1 signaling in the fat body/PG axis is not known yet, but the undergoing systematic analysis of the microbiome should reveal specific bacterial genes that are beneficial in this symbiotic interaction.

\section{Uncoupled Growth and Organ Sparing}

Phenotypic plasticity refers to the ability of a single genotype to generate different phenotypes according to the environmental conditions. This plasticity is based on the differential responses of various tissues to environmental changes. During development, although changes in nutrient availability affect global body size, specific organs can be spared with respect to the rest of the body. Cheng et al. (2011) analyzed the growth response of various organs and tissues under nutrient restriction over the last $36 \mathrm{~h}$ of larval development (not affecting pupariation time). This study revealed that the larval body and endoreplicative tissues (fat body, salivary glands) do not grow during fasting, in contrast to the wing imaginal discs, which retain some growth activity, albeit to a lesser extent than under normal conditions. Finally, the central nervous system appears to be fully spared and to grow to normal size despite restrictive food conditions. Another study, using forced varia- tions in global insulin signaling as a proxy for variations in nutritional conditions, revealed differential responses among imaginal discs, perturbing the normal allometric relationships that exist among organs (Shingleton et al. 2005). For example, whereas the wings and maxillary palps were reduced upon InR inhibition, the genital arches showed limited response. As a consequence, the inhibition of insulin signaling gave rise to animals with larger genitals relative to their wings and overall body size. Insulin sensitivity in different discs depends on the tissue-intrinsic levels of dFOXO activity. Low dFOXO activity in the genital disc could be responsible for minimizing reductions in organ growth on nutrient restriction or reduced insulin signaling in comparison to other organs (Tang et al. 2011).

How can insulin signaling still activate growth in these so-called "critical organs" upon nutrient deprivation when circulating Dilp levels are dramatically decreased? One hint could come from the finding that one of the Dilps, called Dilp6, is produced by the fat body in response to starvation (Slaidina et al. 2009). Up-regulation of Dilp6 by fat cells does not contribute to autocrine activation of IIS, because autophagy remains high in the fat body of starved animals. A deeper analysis revealed the presence of higher intrinsic $\mathrm{dFOXO}$ levels in fat cells than in wing imaginal discs, correlating with the increased sensitivity of this tissue to starvation. In addition, while decreasing Foxo dosage in fat cells increases their sensitivity to starvation, increasing Foxo in wing discs makes them more responsive to nutrient deprivation ( $\mathrm{M}$ Slaidina, $\mathrm{R}$ Delanoue, and $\mathrm{P}$ Léopold, unpubl.). Together, these observations suggest a model whereby, on starvation, Dilp6 produced by fat cells compensates for the reduced secretion of brain Dilps and activates IIS in individual organs or tissues according to their intrinsic levels of dFOXO (Fig. 2).

Another way in which organs can escape starvation-induced growth inhibition is by switching their growth program from nutrient-dependent to nutrient-independent control. In young larvae, brain growth relies on the activities of TOR and InR signaling, and a 
L. Boulan et al.

restricted diet induces a drastic reduction in neuroblast proliferation and brain size. Once a developmental threshold called critical weight (see below) has been passed, however, nutrient restriction no longer delays the transition from larva to pupa and, interestingly, does not affect brain development and growth anymore. Normal brain development and growth is also observed in tor mutants, under conditions of limited amino-acid transport (inactivation of slif), or when systemic insulin levels are reduced. However, in these cases, downstream effectors of these pathways like PI3K/Akt and S6K/4E$\mathrm{BP}$ are still required to control brain growth. Experiments have revealed that another membrane receptor with high sequence similarity to InR, called anaplastic lymphoma kinase (Alk), is highly expressed in the central nervous system and is responsible for activating PI3K signaling and phosphorylating 4E-BP during late larval stages (Cheng et al. 2011). During this period, Alk activity suppresses the requirement for TOR and InR, rendering neuroblast growth and proliferation independent of nutrients. Jeb (Jelly belly), the ligand for Alk, is highly expressed in glial cells, and the specific knockdown of Jeb in glial cells reduces the growth and proliferation of neuroblasts. The glial niche, therefore, provides short-range signaling that protects the neural lineage from nutrient deprivation. Interestingly, this study highlights functional parallels in the strategies used by developing organs and by tumors to evade nutrient limitation. Indeed, activating mutations in ALK have been found in childhood neuroblastomas, and IIS and constitutive PI3K activities have been found to be independent in cases of nutrient resistance in human cancers (Kalaany and Sabatini 2009).

\section{INTERPLAY BETWEEN TISSUE GROWTH AND DEVELOPMENTAL TIMING}

Final body size is determined both by the rate at which the constituting tissues grow and by the duration of their growth period. These two parameters need tight linkage to ensure that organisms reach their proper final size. As shown earlier, these parameters are controlled at the organismal level by hormones, meaning that one prerequisite for understanding the determination of final size is to understand the functional interplay between these hormonal systems.

\section{Critical Weight for Metamorphosis}

Because Drosophila adult size is determined by the extent of growth that has been reached by the end of the larval period, the control of the transition from larva to pupa is critical for size determination. Work on bigger insects like the moth Manduca sexta has paved the way in this area, yielding interesting concepts concerning the control of this transition. In Manduca, a developmental threshold defines the weight at which starvation no longer delays entry into pupal development. Reaching this so-called "critical weight" (CW) constitutes an important but poorly understood transition that occurs soon after ecdysis marking the transition into the last larval instar (Davidowitz et al. 2003). If animals encounter poor nutrient conditions before reaching the CW, their development stalls and subsequent ecdysone pulses are delayed until an appropriate size is reached. Subsequently, when starved larvae are returned to food, pupariation is delayed, giving rise to pupae and adults of normal size. In contrast, once the CW has been passed, metamorphosis can no longer be delayed, and larvae exposed to limiting nutrients therefore develop into small adults. These findings obtained on the Manduca model have been supported by studies on the temporal requirements for insulin signaling in Drosophila. For example, using a temperature-sensitive allele of $\operatorname{In} R$ to switch general IIS on or off at different developmental timepoints, Shingleton et al. (2005) observed a developmental threshold beyond which IIS is no longer required to time the transition into metamorphosis. This threshold is analogous to the CW of Manduca in that it defines a larval size above which nutrition-responding pathways no longer control the timing of metamorphosis. In Drosophila, attempts to define a CW for molting have long been unsuccessful, owing to several differences with the Manduca model. First, the term 
"critical weight" has often been given different meanings, because of the fact that it takes place concomitantly with other developmental checkpoints, like the minimal viable weight (a size below which animals cannot survive complete food withdrawal and never pupariate). Second, when starved above this developmental threshold, Drosophila larvae do not maintain a constant time to pupariation, but rather accelerate their development (Beadle et al. 1938; Stieper et al. 2008). Therefore, starvation modifies the time to pupariation both before and after the CW is reached and cannot be used to determine when the decision to pupariate is made. More recently, a graphical method called the "breakpoint method," based on linear regressions of curves describing the time to pupariation as a function of weight, has been used to define the CW in Drosophila with good accuracy (Stieper et al. 2008). Under standard breeding conditions at $25^{\circ} \mathrm{C}$, the $\mathrm{CW}(\sim 0.9 \mathrm{~g})$ is reached $8 \mathrm{~h}$ after the L2-L3 molt and coincides with a first low titer peak of ecdysone during the L3 instar. However, the mechanism by which this weight threshold is sensed and translated into hormonal outputs remains unknown. In the moth Manduca sexta, before the attainment of the CW, the secretion of PTTH and ecdysone is inhibited by the juvenile hormone ( $\mathrm{JH})$. Once $\mathrm{CW}$ is reached, however, the levels of $\mathrm{JH}$ esterase, an enzyme that inactivates $\mathrm{JH}$ in the hemolymph, increase abruptly. The titers of $\mathrm{JH}$ then decline, allowing the production of PTTH and ecdysone (Browder et al. 2001). In Drosophila, it is known that ablation of $\mathrm{JH}$-producing cells is not sufficient to advance pupariation, nor can exogenous $\mathrm{JH}$ suppress it (Riddiford et al. 2010), indicating that $\mathrm{JH}$ is not involved in timing the onset of metamorphosis.

In Manduca, the CW of each instar is proportional to the initial weight of the same instar, suggesting that the size-sensing mechanism is related to a property set at the beginning of each instar (Nijhout et al. 2006). Interestingly, the volume of the tracheal network increases abruptly at each molt and is not modified among molts (Callier and Nijhout 2011). Although the respiration rate initially increases linearly with body size, it stays constant after larvae have reached the CW. This suggests that the size of the tracheal system that is established during the preceding molt could become limiting during further body mass increase. The CW could therefore correspond to a body mass beyond which the oxygen supply becomes limiting for the tissue respiration rate. Consistent with this possibility, general hypoxia causes a reduction of the $\mathrm{CW}$, although raising larvae in hyperoxic conditions is not sufficient to increase it (Callier and Nijhout 2011). Could a similar hypoxic response be involved in triggering pupariation in Drosophila? Interestingly, low oxygen conditions activate the production of NO (Wingrove and O'Farrell 1999), which is required for pupariation by promoting ecdysone biosynthesis in the ring gland (Cáceres et al. 2011). It is therefore interesting to speculate that, in Drosophila as in Manduca, the CW corresponds to a physiological threshold beyond which the respiration rate becomes limiting, resulting in the turning on of NO production and, consequently, the activation of ecdysone biosynthesis in the PG (Fig. 1). It should be noted that, in both Manduca and Drosophila, the CW is reached long before the larva attains its final size, and in Manduca it occurs even before the imaginal discs have started growing. Therefore, the $\mathrm{CW}$ is not likely to be a reliable indicator of final organ size, but might rather reflect a metabolic/energy threshold that larvae must reach to make it safely through the nonfeeding pupal period.

Insulin Signaling Promotes Ecdysone Synthesis in the Prothoracic Gland

Although the connection between CW and pupariation has not yet been clarified, several studies have highlighted a key role for insulin signaling in the regulation of ecdysone production in the PG (Fig. 3) (Caldwell et al. 2005; Colombani et al. 2005; Mirth et al. 2005). For example, ring gland-specific expression of an activated form of PI3K increases ecdysone levels and leads to animals with reduced size. Conversely, reducing PI3K activity in PG cells inhibits ecdysone production and increases larval growth. Although several groups have reported related ef- 
L. Boulan et al.

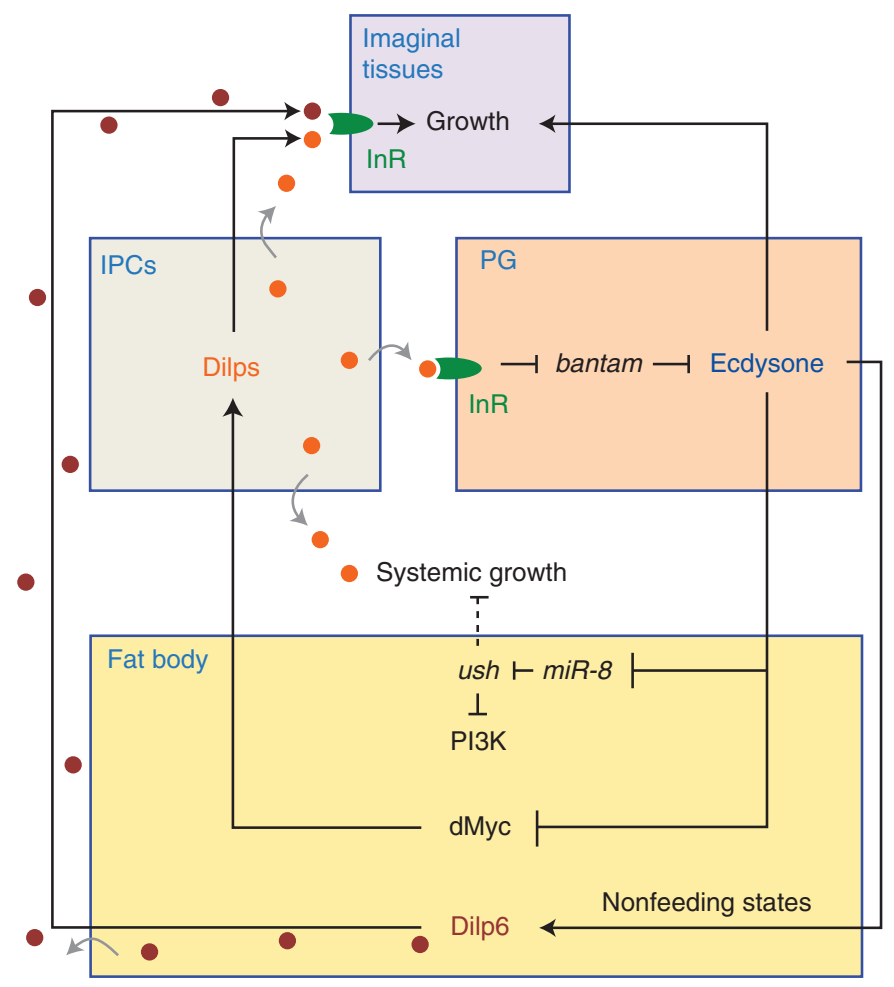

Figure 3. The cross talk between insulin and ecdysone in body growth control. Insulin signaling promotes tissue growth and enhances ecdysone production in the prothoracic gland (PG). Besides its well-described function in developmental transitions, ecdysone has a dual effect on tissue growth. Although it activates autonomous growth of larval tissues through a still unknown mechanism, it inhibits systemic growth by down-regulating dMyc expression in the fat body, resulting in a general inhibition of insulin signaling. The humoral signal produced by fat cells on dMyc activation remains unknown. Ecdysone autonomously antagonizes insulin signaling in fat cells by repressing the growth-promoting microRNA (miRNA) miR-8, whose function is conserved in mammals. miR-8 and its target u-shaped (ush) could also affect systemic growth through another mechanism (dashed line). Finally, the activation of Dilp6 by ecdysone signaling in fat cells could promote specific tissue growth during metamorphosis.

fects on animal size through the manipulation of PI3K activity in the PG, the outcomes have differed depending on the strength of the genetic manipulations involved. Using a weak driver to conduct gain- and loss-of-function experiments in the PG, Colombani et al. (2005) observed variations in basal ecdysone levels with no effects on the peak of ecdysone that occurs before pupariation. Under these conditions, the growth rate of the larvae, but not their developmental timing, was modified. In contrast, stronger transgene expression affects the production of the peak of ecdysone at pupariation, impairing progression into pupal development
(Caldwell et al. 2005; Mirth et al. 2005). These observations indicate that insulin signaling in the PG promotes the production of ecdysone and reduces the final body size, both by lowering the animals' growth rate and by shortening the duration of growth. Surprisingly, a key player in this regulation happens to be the bantam miRNA. In addition to its described role as a cell-autonomous growth inducer, bantam controls systemic growth by repressing basal ecdysone production in the PG. bantam activity in the PG is repressed by IIS, thereby linking IIS and ecdysone production in this organ (Boulan et al. 2013). 
In parallel to its role in promoting ecdysone synthesis, IIS promotes the growth of PG cells. This observation led to a proposal according to which the size of the PG serves as a coupling mechanism between body growth and the timing of metamorphosis (Mirth et al. 2005) —only above a certain size would the PG be competent to produce the peak of ecdysteroids that is required for pupariation. This view is challenged by multiple observations. For example, although overexpressing $\mathrm{dMyc}$ or cyclin $\mathrm{D} / \mathrm{Cdk} 4$ in the PG increases its size, it does not affect the timing of pupariation (Colombani et al. 2005). In addition, overexpressing bantam in the PG leads to larger glands that produce less ecdysone because of the inhibitory role of bantam on ecdysone biosynthesis (Boulan et al. 2013). It, therefore, seems more plausible that IIS activates ecdysone production in the PG independently of its growth-promoting activity. At the same time, modifying IIS in the PG seems to affect the CW (Mirth et al. 2005), suggesting that IIS could participate in translating the assessment of CW into ecdysone production and pupariation.

Rather than participating in size sensing, IIS could coordinate the production of ecdysone with the availability of nutrients in the PG. In line with this idea, limiting food conditions rescues the effects of IIS inhibition in the PG. In the other direction, the body size reduction that is normally induced by forcing expression of IIS in the PG can be rescued by raising animals on rich food (Mirth et al. 2005). What is the physiological role of insulin signaling in this coordination, and how is it linked to the CW? In Bombyx mori, the expression of torso, the receptor for PTTH, is controlled by nutrients (Young et al. 2012). torso is expressed at high levels early in the last instar, and its expression drops when animals reach the CW (Saha et al. 2009). A similar decrease in torso expression at the CW has been observed in Drosophila (McBrayer et al. 2007). torso expression could then rely on insulin signaling until the $\mathrm{CW}$ is reached. Therefore, rather than timing pupariation, the insulin signal could act as a permissive factor for PG cells, making them responsive to PTTH. This possibility is supported by the observation that, after the $\mathrm{CW}$ has been achieved and the endocrine cascade initiated, insulin is no longer required for ecdysone production and metamorphosis (Shingleton et al. 2005).

\section{A Nutrient-Sensing Mechanism in PG Cells}

In parallel to the role played by insulin signaling, an additional mechanism involving TORC1 directly couples nutrient sensing in the PG with ecdysone production and entry into metamorphosis (Fig. 1) (Layalle et al. 2008). Indeed, mild TORC1 inhibition in the PG leads to animal overgrowth as a consequence of prolonged larval development, whereas the growth rate of the animals remains unchanged. This indicates that the activation of TOR signaling is required for the production of ecdysone at the larval/pupal transition, a finding that had been suggested in early experiments measuring the activity of S6kinase, a target of TOR, in purified ring glands of Manduca sexta (Song and Gilbert 1994, 1997). In the other direction, the activation of TOR signaling in the PG rescues ecdysone production as well as the developmental delay observed under poor nutrition. This contrasts with the effects that are observed on growth rates after manipulating IIS in the PG under similar conditions (Colombani et al. 2005). Therefore, although they are closely linked in their actions as growth inducers, the IIS and TOR-signaling pathways participate in distinct physiological responses of PG cells to environmental signals controlling ecdysone production. Under low nutrients, reduced TOR signaling in fat cells leads to low insulin signaling and a reduction in the general growth rate. As a consequence, the CW is reached only after a delay. Subsequently, once the CW has been passed, IIS no longer affects the developmental timing, but the reduced TOR activity in the PG cells prevents ecdysone production, further extending the larval growth period. Intriguingly, complete food withdrawal after the CW accelerates metamorphosis (Beadle et al. 1938; Stieper et al. 2008), indicating that, once the CW has been passed, other mechanisms act to advance pupariation under complete starvation conditions. 
L. Boulan et al.

The Systemic Inhibition of Animal Growth Rates by Ecdysone

A mild inhibition of IIS in the PG reduces the basal levels of ecdysone and leads to bigger larvae (Colombani et al. 2005). This overgrowth is accompanied by a reduction in the expression of ecdysone biosynthesis genes and can be rescued by feeding animals 20E, confirming that the phenotype is caused by reduced ecdysone levels. A close examination of larval growth parameters indicates that the basal levels of ecdysone do not act on the time of pupariation, but rather modulate body growth rate during larval development. Interestingly, general IIS activity is reduced when animals are fed ecdysone (Colombani et al. 2005). This inhibition of IIS can be rescued by silencing the ecdysone receptor $(\mathrm{EcR})$ in fat cells (but not in any other larval tissue), indicating that the main target tissue of ecdysone signaling for systemic growth inhibition is the fat body (Fig. 3). In this tissue, activation of EcR signaling inhibits dMyc activity, a potent activator of systemic growth (Delanoue et al. 2010). The general activation of IIS that is observed upon adipose EcR inhibition suggests that there is an increase in circulating Dilp levels. Indeed, dMyc activity in the fat cells remotely controls Dilp release from the brain IPCs, although the molecular mechanism underlying this control remains unknown (Parisi et al. 2013). Therefore, the activation of ecdysone production by IIS in the PG could be part of a feedback mechanism that permits the integration of nutritional and metabolic inputs from the fat body. With such feedback, the attainment of a storage/metabolic threshold by fat cells would increase Dilp release from the brain by a mechanism involving fat body-derived factors (see above). This, in turn, would activate IIS in PG cells, making them competent to receive the PTTH signal and induce ecdysone production. In turn, high $20 \mathrm{E}$ at the end of larval development would feed back on the fat body, in which it would repress dMyc expression and inhibit systemic growth. Such a mechanism could contribute to the systemic growth arrest that is observed at the end of larval development. Because dMyc also acts downstream of
TOR signaling in the fat body (Teleman et al. 2008), both nutritional signals and ecdysone appear to converge on the regulation of $\mathrm{dMyc}$ in fat body cells to adjust organismal growth in response to both developmental and environmental signals.

Another form of cross talk between ecdysone and insulin signaling involves the miRNA $m i R-8$ (Fig. 3). $m i R-8$ is highly expressed in fat cells, in which it promotes IIS through the inhibition of its target $u$-shaped (ush), thereby influencing body growth rates (Hyun et al. 2009). Interestingly, the human miR-200 family, which is functionally homologous to the Drosophila miR-8, targets FOG2, a vertebrate ortholog of ush. Like USH in Drosophila, FOG2 prevents the activation of PI3K in human cells treated with IGF- 1 by directly binding to $\mathrm{p} 85 \alpha$, the regulatory subunit of PI3K, and by interfering with the formation of a functional PI3K complex. In flies, $m i R-8$ is repressed by ecdysone signaling, and its expression decreases during the third larval instar to reach a minimum level at the larval-pupal transition. Consequently, ush transcripts gradually accumulate during development (Jin et al. 2012). Perturbations of $m i R-8$ and ush expression abolish the ecdysone-mediated inhibition of body growth rates. In addition, the $m i R-8 /$ ush module is required for ecdysone signaling to antagonize insulin signaling. These studies therefore provide a molecular mechanism by which ecdysone can suppress insulin signaling in fat cells. The miR-200 family of miRNAs seems to be also controlled by steroids in mammals, raising the possibility that the regulatory axis of steroid hormone/miR$200 /$ insulin signaling is conserved throughout evolution. However, mild, "physiological" manipulations of IIS in the Drosophila fat body do not affect larval growth rates or pupal size (Colombani et al. 2003; Delanoue et al. 2010). In addition, restoring normal PI3K activity in fat cells does not rescue the size defects observed after manipulating EcR signaling in this tissue (Delanoue et al. 2010). This indicates that, in contrast to dMyc, IIS does not appear to be a relevant target of EcR signaling in fat cells for systemic growth control. This is somehow contradictory with the notion that $m i R-8$ controls 
systemic growth downstream from ecdysone signaling by modulating adipose IIS (Hyun et al. 2009). Therefore, the most plausible role for IIS in the fat body would be to translate nutrient sensing into a general flux of metabolites, by regulating their storage and release from fat cells. Whether adipose IIS also participates in systemic growth control remains under debate.

\section{A Growth/Maturation Checkpoint in Imaginal Tissues that Controls the Timing of Metamorphosis}

Both developmental (maturation of imaginal tissues) and environmental (nutrition) cues feed into ecdysone production and determine the duration of larval growth (Fig. 1). Early experiments established that metamorphosis is delayed when imaginal tissues are damaged by genetic- or X-ray-induced cell death (Russell 1974; Simpson and Schneiderman 1975; Simpson et al. 1980; Parker and Shingleton 2011), or when imaginal disc growth is slowed down by reducing ribosomal protein content (Stieper et al. 2008; Parker and Shingleton 2011). This delay in development allows damaged tissues to regenerate and to grow to the appropriate size after injury. However, the elimination of entire imaginal discs does not alter the timing of metamorphosis (Simpson et al. 1980), suggesting that discs that have not completed a certain amount of growth inhibit the transition to maturation, and that in the absence of discs this transition can be timed by a distinct, redundant feedback mechanism. Importantly, pupariation is also inhibited under mutant conditions in which discs do not stop growing at the end of the larval stage and instead overgrow beyond normal size (Sehnal and Bryant 1993). Both regeneration and overgrowth generate extra cell proliferation, suggesting that proliferating disc cells produce a signal that prevents ecdysone release. Thus, there is a direct relationship between the growth of the imaginal discs and the timing of pupariation. In one study, close examination revealed that damage induced in first or second instar larvae does not delay early molts (Halme et al. 2010). This observation revealed the existence of a developmental checkpoint acting during the third-instar stage, which reflects the varied abilities of damaged discs to regenerate during larval development. Damage inflicted before the checkpoint induces tissue repair, whereas lesions occurring in imaginal tissues after the checkpoint no longer repair and give rise to adults with reduced organ size and patterning defects. $\mathrm{Mu}-$ tations in genes encoding components of the retinoid biosynthetic pathway can partially rescue this damage-induced delay and restore the normal timing of ptth transcription, indicating that retinoids or their metabolites could function as a signal for this mechanism (Halme et al. 2010).

Two recent studies have identified Dilp8, a previously uncharacterized secreted factor related to the ILP family, as a signal produced by growing discs that inhibits entry into metamorphosis (Colombani et al. 2012; Garelli et al. 2012). In the first study, a disc-related neoplastic growth-induced developmental delay was used in a genome-wide screen to identify genes whose disc-specific knockdown could rescue the delay at pupariation. Examining microarrays from various eye tumors, Garelli and colleagues found $\operatorname{dilp} 8$ as a highly enriched gene encoding a secreted peptide. Dilp8 is produced by imaginal disc cells in response to tissue damage or growth perturbation. It is not clear yet how Dilp8 acts to block pupariation. PTTH expression is only slightly delayed by Dilp8 overexpression, although the ecdysone response is abolished. This suggests that Dilp8 could act at the level of PTTH translation, release by PTTH neurons, and/or ecdysone production in PG cells. The elucidation of this mechanism will require the identification of a bona fide Dilp8 receptor. Normal dilp8 mRNA levels drop in mid-third instar, which is likely a prerequisite for the transition to pupal development, as overexpressing dilp8 in imaginal discs is sufficient to delay pupariation and to produce bigger adults. dilp8 loss of function, however, does not lead to early metamorphosis. These observations indicate that the down-regulation of dilp8 expression constitutes a permissive event for the occurrence of metamorphosis, 
L. Boulan et al.

but that the onset of this process requires additional signals.

One striking observation comes from older regeneration and transplantation studies. When growth is impaired in a subset of imaginal discs, intact discs do not generate organs of larger size, even though they benefit from an extended period of growth (Simpson et al. 1980). Therefore, additional mechanisms must be at work, which act to maintain the correct final proportions among organs emerging from damaged or undamaged discs. A recent reevaluation of these early experiments has revealed that unaffected discs are able to align their growth rates to the slower ones (Parker and Shingleton 2011). Under these conditions, animals fed with ecdysone present a reduction of the developmental delay and a disruption of disc growth coordination, suggesting that ecdysone plays a role in this process. Interestingly, ectopic expression of Dilp8 in discs reduces their growth rate, suggesting that Dilp8 could play a role there as well. One possible scenario could be that, on perturbed growth, Dilp8 produced by slow discs inhibits the production of ecdysone, which is needed at basal levels for optimal disc growth (Nijhout and Grunert 2002; Delanoue et al. 2010). As a consequence of the loss of ecdysone, general disc growth would be reduced and coordination could occur. Alternatively, Dilp8 could serve as an endocrine growth inhibitor produced by slow-growing tissues, acting remotely on intact tissues independently of ecdysone. Again, a better understanding of these fascinating coordination mechanisms will benefit from the identification of a Dilp8 receptor and its downstream signaling pathway.

\section{ACKNOWLEDGMENTS}

The authors thank Peter Follette for comments and for proofreading the manuscript. They apologize for uncited work because of space limitations or unintentional omission. They acknowledge the support from the European Research Council (Grant 268813 to L.B. and P.L.) and the Spanish Ministerio de Economía y Competitividad (Grants BFU2010-21123 and CSD2007-00008 to M.M.).

\section{REFERENCES}

Adeghate E, Ponery AS, Wahab A. 2001. Effect of electrical field stimulation on insulin and glucagon secretion from the pancreas of normal and diabetic rats. Horm Metab Res 33: $281-289$.

Alfa RW, Park S, Skelly KR, Poffenberger G, Jain N, Gu X, Kockel L, Wang J, Liu Y, Powers AC, et al. 2015. Suppression of insulin production and secretion by a decretin hormone. Cell Metab 21: 323-333.

Andersen DS, Colombani J, Léopold P. 2013. Coordination of organ growth: Principles and outstanding questions from the world of insects. Trends Cell Biol 23: 336-344.

Arquier N, Géminard C, Bourouis M, Jarretou G, Honegger B, Paix A, Léopold P. 2008. Drosophila ALS regulates growth and metabolism through functional interaction with insulin-like peptides. Cell Metab 7: 333-338.

Arsham AM, Neufeld TP. 2006. Thinking globally and acting locally with TOR. Curr Opin Cell Biol 18: 589-597.

Aspinwall CA, Lakey JR, Kennedy RT. 1999. Insulin-stimulated insulin secretion in single pancreatic $\beta$ cells. J Biol Chem 274: 6360-6365.

Avruch J, Long X, Ortiz-Vega S, Rapley J, Papageorgiou A, Dai N. 2009. Amino acid regulation of TOR complex 1. Am J Physiol Endocrinol Metab 296: E592-E602.

Baehrecke EH. 2000. Steroid regulation of programmed cell death during Drosophila development. Cell Death Differ 7: 1057-1062.

Bai H, Kang P, Tatar M. 2012. Drosophila insulin-like peptide-6 (dilp6) expression from fat body extends lifespan and represses secretion of Drosophila insulin-like peptide-2 from the brain. Aging Cell 11: 978-985.

Beadle GW, Tatum EL, Clancy C. 1938. Food level in relation to rate of development and eye pigmentation in Drosophila melanogaster. Biol Bull 75: 447-462.

Birse RT, Söderberg JAE, Luo J, Winther AME, Nässel DR. 2011. Regulation of insulin-producing cells in the adult Drosophila brain via the tachykinin peptide receptor DTKR. J Exp Biol 214: 4201-4208.

Bjordal M, Arquier N, Kniazeff J, Pin JP, Léopold P. 2014. Sensing of amino acids in a dopaminergic circuitry promotes rejection of an incomplete diet in Drosophila. Cell 156: $510-521$.

Böhni R, Riesgo-Escovar J, Oldham S, Brogiolo W, Stocker H, Andruss BF, Beckingham K, Hafen E. 1999. Autonomous control of cell and organ size by CHICO, a Drosophila homolog of vertebrate IRS1-4. Cell 97: 865-875.

Boulan L, Martín D, Milán M. 2013. bantam miRNA promotes systemic growth by connecting insulin signaling and ecdysone production. Curr Biol 23: 473-478.

Brogiolo W, Stocker H, Ikeya T, Rintelen F, Fernandez R, Hafen E. 2001. An evolutionarily conserved function of the Drosophila insulin receptor and insulin-like peptides in growth control. Curr Biol 11: 213-221.

Broughton S, Alic N, Slack C, Bass T, Ikeya T, Vinti G, Tommasi AM, Driege Y, Hafen E, Partridge L. 2008. Reduction of DILP2 in Drosophila triages a metabolic phenotype from lifespan revealing redundancy and compensation among DILPs. PLoS ONE 3: e3721. 
Browder MH, D'Amico LJ, Nijhout HF. 2001. The role of low levels of juvenile hormone esterase in the metamorphosis of Manduca sexta. J Insect Sci 1: 11.

Bryk B, Hahn K, Cohen SM, Teleman AA. 2010. MAP4K3 regulates body size and metabolism in Drosophila. Dev Biol 344: 150-157.

Cáceres L, Necakov AS, Schwartz C, Kimber S, Roberts IJH, Krause HM. 2011. Nitric oxide coordinates metabolism, growth, and development via the nuclear receptor E75. Genes Dev 25: 1476-1485.

Caldwell PE, Walkiewicz M, Stern M. 2005. Ras activity in the Drosophila prothoracic gland regulates body size and developmental rate via ecdysone release. Curr Biol 15: 1785-1795.

Callier V, Nijhout HF. 2011. Control of body size by oxygen supply reveals size-dependent and size-independent mechanisms of molting and metamorphosis. Proc Natl Acad Sci 108: 14664-14669.

Callier V, Shingleton AW, Brent CS, Ghosh SM, Kim J, Harrison JF. 2013. The role of reduced oxygen in the developmental physiology of growth and metamorphosis initiation in Drosophila melanogaster. J Exp Biol 216: 4334 4340.

Cheetham SW, Brand AH. 2013. Insulin finds its niche. Science 340: 817-818.

Chell JM, Brand AH. 2010. Nutrition-responsive glia control exit of neural stem cells from quiescence. Cell 143: 1161-1173.

Cheng LY, Bailey AP, Leevers SJ, Ragan TJ, Driscoll PC, Gould AP. 2011. Anaplastic lymphoma kinase spares organ growth during nutrient restriction in Drosophila. Cell 146: $435-447$.

Colombani J, Raisin S, Pantalacci S, Radimerski T, Montagne J, Leopold P. 2003. A nutrient sensor mechanism controls Drosophila growth. Cell 114: 739-749.

Colombani J, Bianchini L, Layalle S, Pondeville E, DauphinVillemant C, Antoniewski C, Carre C, Noselli S, Leopold P. 2005. Antagonistic actions of ecdysone and insulins determine final size in Drosophila. Science 310: 667-670.

Colombani J, Andersen DS, Léopold P. 2012. Secreted peptide Dilp8 coordinates Drosophila tissue growth with developmental timing. Science 336: 582-585.

Crocker A, Shahidullah M, Levitan IB, Sehgal A. 2010. Identification of a neural circuit that underlies the effects of octopamine on sleep:wake behavior. Neuron 65: 670681.

Davidowitz G, D’Amico LJ, Nijhout HF. 2003. Critical weight in the development of insect body size. Evol Dev 5: 188-197.

D'Avino PP, Thummel CS. 2000. The ecdysone regulatory pathway controls wing morphogenesis and integrin expression during Drosophila metamorphosis. Dev Biol 220: 211-224.

Delanoue R, Slaidina M, Leopold P. 2010. The steroid hormone ecdysone controls systemic growth by repressing dMyc function in Drosophila fat cells. Dev Cell 18: 1012-1021.

Demontis F, Perrimon N. 2009. Integration of insulin receptor/Foxo signaling and $\mathrm{dMyc}$ activity during muscle growth regulates body size in Drosophila. Development 136: $983-993$.
Demontis F, Patel VK, Swindell WR, Perrimon N. 2014. Intertissue control of the nucleolus via a myokine-dependent longevity pathway. Cell Rep 7: 1481-1494.

De Virgilio C, Loewith R. 2006. The TOR signalling network from yeast to man. Int J Biochem Cell Biol 38: 1476-1481.

Domené HM, Hwa V, Argente J, Wit JM, Wit JM, CamachoHübner C, Jasper HG, Pozo J, van Duyvenvoorde HA, Yakar S, et al. 2009. Human acid-labile subunit deficiency: Clinical, endocrine and metabolic consequences. Horm Res 72: 129-141.

Dong J, Pan D. 2004. Tsc2 is not a critical target of Akt during normal Drosophila development. Genes Dev 18: 2479-2484.

Dong H, Kumar M, Zhang Y, Gyulkhandanyan A, Xiang YY, Ye B, Perrella J, Hyder A, Zhang N, Wheeler M, et al. 2006. $\gamma$-Aminobutyric acid up- and downregulates insulin secretion from $\beta$ cells in concert with changes in glucose concentration. Diabetologia 49: 697-705.

Drucker DJ, Philippe J, Mojsov S, Chick WL, Habener JF. 1987. Glucagon-like peptide I stimulates insulin gene expression and increases cyclic AMP levels in a rat islet cell line. Proc Natl Acad Sci 84: 3434-3438.

Duan C, Xu Q. 2005. Roles of insulin-like growth factor (IGF) binding proteins in regulating IGF actions. Gen Comp Endocrinol 142: 44-52.

Duran A, Amanchy R, Linares JF, Joshi J, Abu-Baker S, Porollo A, Hansen M, Moscat J, Diaz-Meco MT. 2011.p62 is a key regulator of nutrient sensing in the mTORC1 pathway. Mol Cell 44: 134-146.

Edgar BA. 2006. How flies get their size: Genetics meets physiology. Nat Rev Genet 7: 907-916.

Enell LE, Kapan N, Söderberg JAE, Kahsai L, Nässe DR. 2010. Insulin signaling, lifespan and stress resistance are modulated by metabotropic GABA receptors on insulin producing cells in the brain of Drosophila. PLOS ONE 5: e15780.

Figueroa-Clarevega A, Bilder D. 2015. Malignant Drosophila tumors interrupt insulin signaling to induce cachexialike wasting. Dev Cell 33: 47-55.

Francis VA, Zorzano A, Teleman AA. 2010. dDOR is an EcR coactivator that forms a feed-forward loop connecting insulin and ecdysone signaling. Curr Biol 20: 1799-808.

Fridell Y-WC, Hoh M, Kréneisz O, Hosier S, Chang C, Scantling D, Mulkey DK, Helfand SL. 2009. Increased uncoupling protein (UCP) activity in Drosophila insulin-producing neurons attenuates insulin signaling and extends lifespan. Aging (Albany NY) 1: 699-713.

Garelli A, Gontijo AM, Miguela V, Caparros E, Dominguez M. 2012. Imaginal discs secrete insulin-like peptide 8 to mediate plasticity of growth and maturation. Science 336: 579-582.

Garofalo RS. 2002. Genetic analysis of insulin signaling in Drosophila. Trends Endocrinol Metab 13: 156-162.

Géminard C, Rulifson EJ, Léopold P. 2009. Remote control of insulin secretion by fat cells in Drosophila. Cell Metab 10: 199-207.

Gibbens YY, Warren JT, Gilbert LI, O'Connor MB. 2011. Neuroendocrine regulation of Drosophila metamorphosis requires TGF- $\beta$ /activin signaling. Development 138: 2693-2703. 
L. Boulan et al.

Gilbert LI. 2004. Halloween genes encode P450 enzymes that mediate steroid hormone biosynthesis in Drosophila melanogaster. Mol Cell Endocrinol 215: 1-10.

Goberdhan DCI, Paricio N, Goodman EC, Mlodzik M, Wilson C. 1999. Drosophila tumor suppressor PTEN controls cell size and number by antagonizing the Chico/PI3-kinase signaling pathway. Genes Dev 13: 3244-3258.

Grewal SS, Li L, Orian A, Eisenman RN, Edgar BA. 2005. Myc-dependent regulation of ribosomal RNA synthesis during Drosophila development. Nat Cell Biol 7: $295-$ 302.

Grönke S, Clarke D-F, Broughton S, Andrews TD, Partridge L. 2010. Molecular evolution and functional characterization of Drosophila insulin-like peptides. PLoS Genet 6: e1000857.

Guertin DA, Guntur KVP, Bell GW, Thoreen CC, Sabatini DM. 2006. Functional genomics identifies TOR-regulated genes that control growth and division. Curr Biol 16: 958-970.

Guittard E, Blais C, Maria A, Parvy J-P, Pasricha S, Lumb C, Lafont R, Daborn PJ, Dauphin-Villemant C. 2011. CYP18A1, a key enzyme of Drosophila steroid hormone inactivation, is essential for metamorphosis. Dev Biol 349: $35-45$.

Halme A, Cheng M, Hariharan IK. 2010. Retinoids regulate a developmental checkpoint for tissue regeneration in Drosophila. Curr Biol 20: 458-463.

Hardie DG. 2007. AMP-activated/SNF1 protein kinases: Conserved guardians of cellular energy. Nat Rev Mol Cell Biol 8: 774-785.

Holz MK, Ballif BA, Gygi SP, Blenis J. 2005. mTOR and S6K1 mediate assembly of the translation preinitiation complex through dynamic protein interchange and ordered phosphorylation events. Cell 123: 569-580.

Honegger B, Galic M, Köhler K, Wittwer F, Brogiolo W, Hafen E, Stocker H. 2008. Imp-L2, a putative homolog of vertebrate IGF-binding protein 7, counteracts insulin signaling in Drosophila and is essential for starvation resistance. J Biol 7: 10.

Huang X, Suyama K, Buchanan J, Zhu AJ, Scott MP. 2005. A Drosophila model of the Niemann-Pick type C lysosome storage disease: $\operatorname{dnpcla}$ is required for molting and sterol homeostasis. Development 132: 5115-5124.

Huang X, Warren JT, Buchanan J, Gilbert LI, Scott MP. 2007. Drosophila Niemann-Pick type C-2 genes control sterol homeostasis and steroid biosynthesis: A model of human neurodegenerative disease. Development 134: 37333742.

Huang X, Warren JT, Gilbert LI. 2008. New players in the regulation of ecdysone biosynthesis. J Genet Genomics 35: $1-10$.

Hyun S, Lee JH, Jin H, Nam J, Namkoong B, Lee G, Chung J, Kim VN. 2009. Conserved microRNA miR-8/miR-200 and its target USH/FOG2 control growth by regulating PI3K. Cell 139: 1096-1108.

Ikeya T, Galic M, Belawat P, Nairz K, Hafen E. 2002. Nutrient-dependent expression of insulin-like peptides from neuroendocrine cells in the CNS contributes to growth regulation in Drosophila. Curr Biol 12: 1293-1300.
Inoki K, Li Y, Zhu T, Wu J, Guan K-L. 2002. TSC2 is phosphorylated and inhibited by Akt and suppresses mTOR signalling. Nat Cell Biol 4: 648-657.

Inoki K, Li Y, Xu T, Guan K-L. 2003a. Rheb GTPase is a direct target of TSC2 GAP activity and regulates mTOR signaling. Genes Dev 17: 1829-1834.

Inoki K, Zhu T, Guan K-L. 2003b. TSC2 mediates cellular energy response to control cell growth and survival. Cell 115: $577-590$.

Jin H, Kim V, Hyun S. 2012. Conserved microRNA miR-8 controls body size in response to steroid signaling in Drosophila. Genes Dev 26: 1427-1432.

Johnston DM, Sedkov Y, Petruk S, Riley KM, Fujioka M, Jaynes JB, Mazo A. 2011. Ecdysone- and NO-mediated gene regulation by competing EcR/Usp and E75A nuclear receptors during Drosophila development. Mol Cell 44: $51-61$.

Kalaany NY, Sabatini DM. 2009. Tumours with PI3K activation are resistant to dietary restriction. Nature 458: 725-731.

Kaplan DD, Zimmermann G, Suyama K, Meyer T, Scott MP. 2008. A nucleostemin family GTPase, NS3, acts in serotonergic neurons to regulate insulin signaling and control body size. Genes Dev 22: 1877-1893.

Kawakami A, Kataoka H, Oka T, Mizoguchi A, KimuraKawakami M, Adachi T, Iwami M, Nagasawa H, Suzuki A, Ishizaki H. 1990. Molecular cloning of the Bombyx mori prothoracicotropic hormone. Science 247: 13331335.

Killip LE, Grewal SS. 2012. DREF is required for cell and organismal growth in Drosophila and functions downstream of the nutrition/TOR pathway. Dev Biol 371: 191-202.

Kim SK, Rulifson EJ. 2004. Conserved mechanisms of glucose sensing and regulation by Drosophila corpora cardiaca cells. Nature 431: 316-320.

Kim E, Goraksha-Hicks P, Li L, Neufeld TP, Guan K-L. 2008. Regulation of TORC1 by Rag GTPases in nutrient response. Nat Cell Biol 10: 935-945.

Kim Y-M, Stone M, Hwang TH, Kim Y-G, Dunlevy JR, Griffin TJ, Kim D-H. 2012. SH3BP4 is a negative regulator of amino acid-Rag GTPase-mTORC1 signaling. $\mathrm{Mol}$ Cell 46: 1-14.

King-Jones K, Thummel CS. 2005. Nuclear receptors-A perspective from Drosophila. Nat Rev Genet 6: 311-323.

Kréneisz O, Chen X, Fridell Y-WC, Mulkey DK. 2010. Glucose increases activity and $\mathrm{Ca}^{2+}$ in insulin-producing cells of adult Drosophila. Neuroreport 21: 1116-1120.

Kwon Y, Song W, Droujinine IA, Hu Y, Asara JM, Perrimon N. 2015. Systemic organ wasting induced by localized expression of the secreted insulin/IGF antagonist ImpL2. Dev Cell 33: 36-46.

Layalle S, Arquier N, Léopold P. 2008. The TOR pathway couples nutrition and developmental timing in Drosophila. Dev Cell 15: 568-577.

Lee CY, Baehrecke EH. 2001. Steroid regulation of autophagic programmed cell death during development. Development 128: 1443-1455.

Lee KS, Kwon OY, Lee JH, Kwon K, Min KJ, Jung SA, Kim AK, You KH, Tatar M, Yu K. 2008. Drosophila short neu- 
ropeptide $\mathrm{F}$ signalling regulates growth by ERK-mediated insulin signalling. Nat Cell Biol 10: 468-475.

Leevers SJ, Weinkove D, MacDougall LK, Hafen E, Waterfield MD. 1996. The Drosophila phosphoinositide-3-kinase Dp110 promotes cell growth. EMBO J 15: 65846594.

Loewith R, Jacinto E, Wullschleger S, Lorberg A, Crespo JL, Bonenfant D, Oppliger W, Jenoe P, Hall MN. 2002. Two TOR complexes, only one of which is rapamycin sensitive, have distinct roles in cell growth control. Mol Cell 10: 457-468.

Luo J, Becnel J, Nichols CD, Nässel DR. 2012. Insulin-producing cells in the brain of adult Drosophila are regulated by the serotonin 5-HT1A receptor. Cell Mol Life Sci 69: $471-484$.

Luo J, Lushchak OV, Goergen P, Williams MJ, Nässel DR. 2014. Drosophila insulin-producing cells are differentially modulated by serotonin and octopamine receptors and affect social behavior. PLoS ONE 9: e99732.

Marshall L, Rideout EJ, Grewal SS. 2012. Nutrient/TORdependent regulation of RNA polymerase III controls tissue and organismal growth in Drosophila. EMBO J 31: 1916-1930.

McBrayer Z, Ono H, Shimell M, Parvy JP, Beckstead RB, Warren JT, Thummel CS, Dauphin-Villemant C, Gilbert LI, O'Connor MB. 2007. Prothoracicotropic hormone regulates developmental timing and body size in Drosophila. Dev Cell 13: 857-871.

McBride A, Ghilagaber S, Nikolaev A, Hardie DG. 2009. The glycogen-binding domain on the AMPK $\beta$ subunit allows the kinase to act as a glycogen sensor. Cell Metab 9: 23-34.

McIntyre N, Holdsworth C, Turner D. 1964. New interpretation of oral glucose tolerance. Lancet 4: 20-21.

Miguel-Aliaga I. 2012. Nerveless and gutsy: Intestinal nutrient sensing from invertebrates to humans. Semin Cell Dev Biol 23: 614-620.

Miron M, Verdu J, Lachance PE, Birnbaum MJ, Lasko PF, Sonenberg N. 2001. The translational inhibitor 4E-BP is an effector of PI3K/Akt signalling and cell growth in Drosophila. Nat Cell Biol 3: 596-601.

Mirth CK, Riddiford LM. 2007. Size assessment and growth control: How adult size is determined in insects. Bioessays 29: 344-355.

Mirth CK, Shingleton AW. 2012. Integrating body and organ size in Drosophila: Recent advances and outstanding problems. Front Endocrinol (Lausanne) 3: 1-13.

Mirth C, Truman JW, Riddiford LM. 2005. The role of the prothoracic gland in determining critical weight for metamorphosis in Drosophila melanogaster. Curr Biol 15: 1796-1807.

Miyamoto T, Slone J, Song X, Amrein H. 2012. A fructose receptor functions as a nutrient sensor in the Drosophila brain. Cell 151: 1113-1125.

Nakae J, Kido Y, Accili D. 2001. Distinct and overlapping functions of insulin and IGF-I receptors. Endocr Rev 22: $818-835$.

Nijhout HF, Grunert LW. 2002. Bombyxin is a growth factor for wing imaginal disks in Lepidoptera. Proc Natl Acad Sci 99: 15446-15450.
Nijhout H, Davidowitz G, Roff D. 2006. A quantitative analysis of the mechanism that controls body size in Manduca sexta. J Biol 5: 16.

Okamoto N, Yamanaka N, Yagi Y, Nishida Y, Kataoka H, O'Connor MB, Mizoguchi A. 2009. A fat body-derived IGF-like peptide regulates postfeeding growth in Drosophila. Dev Cell 17: 885-891.

Okamoto N, Nakamori R, Murai T, Yamauchi Y, Masuda A, Nishimura T. 2013. A secreted decoy of InR antagonizes insulin/IGF signaling to restrict body growth in Drosophila. Genes Dev 27: 87-97.

Ou Q, Magico A, King-Jones K. 2011. Nuclear receptor DHR4 controls the timing of steroid hormone pulses during Drosophila development. PLoS Biol 9: e1001160.

Pallares-Cartes C, Cakan-Akdogan G, Teleman Aa. 2012. Tissue-specific coupling between insulin/IGF and TORC1 signaling via PRAS40 in Drosophila. Dev Cell 22: $172-182$.

Parisi F, Riccardo S, Zola S, Lora C, Grifoni D, Brown LM, Bellosta P. 2013. dMyc expression in the fat body affects DILP2 release and increases the expression of the fat desaturase Desat1 resulting in organismal growth. Dev Biol 379: 64-75.

Park S, Alfa RW, Topper SM, Kim GES, Kockel L, Kim SK. 2014. A genetic strategy to measure circulating Drosophila insulin reveals genes regulating insulin production and secretion. PLoS Genet 10: e1004555.

Parker NF, Shingleton AW. 2011. The coordination of growth among Drosophila organs in response to localized growth-perturbation. Dev Biol 357: 318-325.

Pedersen BK, Febbraio MA. 2012. Muscles, exercise and obesity: Skeletal muscle as a secretory organ. Nat Rev Endocrinol 8: 457-465.

Petryk A, Warren JT, Marqués G, Jarcho MP, Gilbert LI, Kahler J, Parvy J-P, Li Y, Dauphin-Villemant C, O'Connor MB. 2003. Shade is the Drosophila P450 enzyme that mediates the hydroxylation of ecdysone to the steroid insect molting hormone 20-hydroxyecdysone. Proc Natl Acad Sci 100: 13773-13778.

Potter CJ, Huang H, Xu T. 2001. Drosophila Tscl functions with Tsc2 to antagonize insulin signaling in regulating cell growth, cell proliferation, and organ size. Cell 105: 357-368.

Potter CJ, Pedraza LG, Xu T. 2002. Akt regulates growth by directly phosphorylating Tsc2. Nat Cell Biol 4: 658-665.

Rajan A, Perrimon N. 2012. Drosophila cytokine unpaired 2 regulates physiological homeostasis by remotely controlling insulin secretion. Cell 151: 123-137.

Reinking J, Lam MMS, Pardee K, Sampson HM, Liu S, Yang P, Williams S, White W, Lajoie G, Edwards A, et al. 2005. The Drosophila nuclear receptor e75 contains heme and is gas responsive. Cell 122: 195-207.

Rewitz KF, Yamanaka N, Gilbert LI, O’Connor MB. 2009. The insect neuropeptide PTTH activates receptor tyrosine kinase torso to initiate metamorphosis. Science 326: 1403-1405.

Rewitz KF, Yamanaka N, O’Connor MB. 2010. Steroid hormone inactivation is required during the juvenile-adult transition in Drosophila. Dev Cell 19: 895-902. 
L. Boulan et al.

Ribeiro C, Dickson BJ. 2010. Sex peptide receptor and neuronal TOR/S6K signaling modulate nutrient balancing in Drosophila. Curr Biol 20: 1000-1005.

Riddiford LM. 1993. Hormone receptors and the regulation of insect metamorphosis. Receptor 3: 203-209.

Riddiford LM, Truman JW, Mirth CK, Shen Y-C. 2010. A role for juvenile hormone in the prepupal development of Drosophila melanogaster. Development 137: $1117-$ 1126.

Rideout EJ, Marshall L, Grewal SS. 2012. Drosophila RNA polymerase III repressor Maf1 controls body size and developmental timing by modulating tRNAiMet synthesis and systemic insulin signaling. Proc Natl Acad Sci 109: 1139-1144.

Rodenfels J, Lavrynenko O, Ayciriex S, Sampaio JL, Carvalho M, Shevchenko A, Eaton S. 2014. Production of systemically circulating Hedgehog by the intestine couples nutrition to growth and development. Genes Dev 28: 2636-2651.

Rulifson EJ, Kim SK, Nusse R. 2002. Ablation of insulinproducing neurons in flies: Growth and diabetic phenotypes. Science 296: 1118-1120.

Russell M. 1974. Pattern formation in the imaginal discs of a temperature sensitive cell-lethal mutant of Drosophila melanogaster. Dev Biol 40: 24-39.

Rusten TE, Lindmo K, Juhász G, Sass M, Seglen PO, Brech A, Stenmark H. 2004. Programmed autophagy in the Drosophila fat body is induced by ecdysone through regulation of the PI3K pathway. Dev Cell 7: 179-192.

Saha AK, Chaudhuri AB, Moorthy SM, Roy S. 2009. Studies on determination of larval critical weight in silkworm, Bombyx mori L.-An index of quality. Int J Ind Entomol 19: $243-248$.

Sancak Y, Thoreen CC, Peterson TR, Lindquist RA, Kang SA, Spooner E, Carr Sa, Sabatini DM. 2007. PRAS40 is an insulin-regulated inhibitor of the mTORC1 protein kinase. Mol Cell 25: 903-915.

Sancak Y, Peterson TR, Shaul YD, Lindquist RA, Thoreen CC, Bar-Peled L, Sabatini DM. 2008. The Rag GTPases bind raptor and mediate amino acid signaling to mTORC1. Science 320: 1496-1501.

Sancak Y, Bar-Peled L, Zoncu R, Markhard AL, Nada S, Sabatini DM. 2010. Ragulator-Rag complex targets mTORC1 to the lysosomal surface and is necessary for its activation by amino acids. Cell 141: 290-303.

Saucedo LJ, Gao X, Chiarelli DA, Li L, Pan D, Edgar BA. 2003. Rheb promotes cell growth as a component of the insulin/TOR signalling network. Nat Cell Biol 5: 566571.

Schleich S, Teleman Aa. 2009. Akt phosphorylates both Tscl and Tsc2 in Drosophila, but neither phosphorylation is required for normal animal growth. PLoS ONE 4: e6305.

Schubiger M, Truman JW. 2000. The RXR ortholog USP suppresses early metamorphic processes in Drosophila in the absence of ecdysteroids. Development 127: 11511159.

Schubiger M, Carré C, Antoniewski C, Truman JW. 2005. Ligand-dependent de-repression via EcR/USP acts as a gate to coordinate the differentiation of sensory neurons in the Drosophila wing. Development 132: 5239-5248.
Scott RC, Schuldiner O, Neufeld TP. 2004. Role and regulation of starvation-induced autophagy in the Drosophila fat body. Dev Cell 7: 167-178.

Sehnal F, Bryant PJ. 1993. Delayed pupariation in Drosophila imaginal disc overgrowth mutants is associated with reduced ecdysteroid titer. J Insect Physiol 39: 1051-1059.

Shin SC, Kim S-H, You H, Kim B, Kim AC, Lee K-A, Yoon JH, Ryu J-H, Lee W-J. 2011. Drosophila microbiome modulates host developmental and metabolic homeostasis via insulin signaling. Science 334: 670-674.

Shingleton AW, Das J, Vinicius L, Stern DL. 2005. The temporal requirements for insulin signaling during development in Drosophila. PLoS Biol 3: e289.

Simpson P, Schneiderman HA. 1975. Isolation of temperature sensitive mutations blocking clone development in Drosophila melanogaster, and the effects of a temperature sensitive cell lethal mutation on pattern formation in imaginal discs. Wilhelm Roux's Arch 178: 247-275.

Simpson P, Berreur P, Berreur-Bonnenfant J. 1980. The initiation of pupariation in Drosophila: Dependence on growth of the imaginal discs. J Embryol Exp Morphol 57: 155-165.

Slaidina M, Delanoue R, Gronke S, Partridge L, Léopold P. 2009. A Drosophila insulin-like peptide promotes growth during nonfeeding states. Dev Cell 17: 874-84.

Song Q, Gilbert LI. 1994. S6 phosphorylation results from prothoracicotropic hormone stimulation of insect prothoracic glands: A role for S6 kinase. Dev Genet 15: 332 338.

Song Q, Gilbert LI. 1997. Molecular cloning, developmental expression, and phosphorylation of ribosomal protein S6 in the endocrine gland responsible for insect molting. $J$ Biol Chem 272: 4429-4435.

Sonoda N, Imamura T, Yoshizaki T, Babendure JL, Lu J-C, Olefsky JM. 2008. $\beta$-Arrestin-1 mediates glucagon-like peptide-1 signaling to insulin secretion in cultured pancreatic $\beta$ cells. Proc Natl Acad Sci 105: 6614-6619.

Sousa-Nunes R, Yee LL, Gould AP. 2011. Fat cells reactivate quiescent neuroblasts via TOR and glial insulin relays in Drosophila. Nature 471: 508-512.

Spéder P, Brand AH. 2014. Gap junction proteins in the blood-brain barrier control nutrient-dependent reactivation of Drosophila neural stem cells. Dev Cell 30: 309321.

Stieper BC, Kupershtok M, Driscoll MV, Shingleton AW. 2008. Imaginal discs regulate developmental timing in Drosophila melanogaster. Dev Biol 321: 18-26.

Stocker H, Radimerski T, Schindelholz B, Wittwer F, Belawat P, Daram P, Breuer S, Thomas G, Hafen E. 2003. Rheb is an essential regulator of $S 6 \mathrm{~K}$ in controlling cell growth in Drosophila. Nat Cell Biol 5: 559-565.

Storelli G, Defaye A, Erkosar B, Hols P, Royet J, Leulier F. 2011. Lactobacillus plantarum promotes Drosophila systemic growth by modulating hormonal signals through TOR-dependent nutrient sensing. Cell Metab 14: $403-$ 414.

Talamillo A, Sánchez J, Cantera R, Pérez C, Martín D, Caminero E, Barrio R. 2008. Smt3 is required for Drosophila melanogaster metamorphosis. Development 135: 16591668. 
Talamillo A, Herboso L, Pirone L, Pérez C, González M, Sánchez J, Mayor U, Lopitz-Otsoa F, Rodriguez MS, Sutherland JD, et al. 2013. Scavenger receptors mediate the role of SUMO and Ftz-f1 in Drosophila steroidogenesis. PLoS Genet 9: e1003473.

Talbot WS, Swyryd EA, Hogness DS. 1993. Drosophila tissues with different metamorphic responses to ecdysone express different ecdysone receptor isoforms. Cell 73: 1323-1337.

Tang HY, Smith-Caldas MSB, Driscoll MV, Salhadar S, Shingleton AW. 2011. FOXO regulates organ-specific phenotypic plasticity in Drosophila. PLoS Genet 7: e1002373.

Tapon N, Ito N, Dickson BJ, Treisman JE, Hariharan IK. 2001. The Drosophila tuberous sclerosis complex gene homologs restrict cell growth and cell proliferation. Cell 105: 345-355.

Tee AR, Manning BD, Roux PP, Cantley LC, Blenis J. 2003. Tuberous sclerosis complex gene products, Tuberin and Hamartin, control mTOR signaling by acting as a GTPase-activating protein complex toward Rheb. Curr Biol 13: 1259-1268.

Teleman AA, Chen YW, Cohen SM. 2005. 4E-BP functions as a metabolic brake used under stress conditions but not during normal growth. Genes Dev 19: 1844-1848.

Teleman AA, Hietakangas V, Sayadian AC, Cohen SM. 2008. Nutritional control of protein biosynthetic capacity by insulin via Myc in Drosophila. Cell Metab 7: 21-32.

Thomas HE, Stunnenberg HG, Stewart AF. 1993. Heterodimerization of the Drosophila ecdysone receptor with retinoid X receptor and ultraspiracle. Nature 362: 471-475.

Toshima N, Tanimura T. 2012. Taste preference for amino acids is dependent on internal nutritional state in Drosophila melanogaster. J Exp Biol 215: 2827-2832.

Vander Haar E, Lee S-I, Bandhakavi S, Griffin TJ, Kim D-H. 2007. Insulin signalling to mTOR mediated by the Akt/ PKB substrate PRAS40. Nat Cell Biol 9: 316-323.

Vargas MA, Luo N, Yamaguchi A, Kapahi P. 2010. A role for S6 kinase and serotonin in postmating dietary switch and balance of nutrients in D. melanogaster. Curr Biol 20: 1113-1125.

Varghese J, Lim SF, Cohen SM. 2010. Drosophila miR-14 regulates insulin production and metabolism through its target, sugarbabe. Genes Dev 24: 2748-2753.

Wang X, Li W, Williams M, Terada N, Alessi DR, Proud CG. 2001. Regulation of elongation factor 2 kinase by p90(RSK1) and p70 S6 kinase. Eur Mol Biol Organ J 20: 4370-4379.

Wang S, Tulina N, Carlin DL, Rulifson EJ. 2007. The origin of islet-like cells in Drosophila identifies parallels to the vertebrate endocrine axis. Proc Natl Acad Sci 104: $19873-$ 19878.

Warren JT, Yerushalmi Y, Shimell MJ, O'Connor MB, Restifo LL, Gilbert LI. 2006. Discrete pulses of molting hormone, 20-hydroxyecdysone, during late larval development of Drosophila melanogaster: Correlations with changes in gene activity. Dev Dyn 235: 315-326.

Wingrove JA, O'Farrell PH. 1999. Nitric oxide contributes to behavioral, cellular, and developmental responses to low oxygen in Drosophila. Cell 98: 105-114.

Wollam J, Antebi A. 2011. Sterol regulation of metabolism, homeostasis, and development. Annu Rev Biochem 80: 885-916.

Wu Q, Brown MR. 2006. Signaling and function of insulinlike peptides in insects. Annu Rev Entomol 51: 1-24.

Wullschleger S, Loewith R, Hall MN. 2006. TOR signaling in growth and metabolism. Cell 124: 471-484.

Xiang Y, Liu Z, Huang X. 2010. br regulates the expression of the ecdysone biosynthesis gene npc1. Dev Biol 344: 800808.

Yamaguchi T, Fernandez R, Roth RA. 1995. Comparison of the signaling abilities of the Drosophila and human insulin receptors in mammalian cells. Biochemistry 34: 4962 4968.

Yamanaka N, Rewitz KF, O’Connor MB. 2013. Ecdysone control of developmental transitions: Lessons from Drosophila research. Annu Rev Entomol 58: 497-516.

Yao TP, Forman BM, Jiang Z, Cherbas L, Chen JD, McKeown M, Cherbas P, Evans RM. 1993. Functional ecdysone receptor is the product of EcR and Ultraspiracle genes. $\mathrm{Na}$ ture 366: $476-479$.

Young S-C, Yeh W-L, Gu S-H. 2012. Transcriptional regulation of the PTTH receptor in prothoracic glands of the silkworm, Bombyx mori. J Insect Physiol 58: 102-109.

Zhang H, Stallock JP, Ng JC, Reinhard C, Neufeld TP. 2000. Regulation of cellular growth by the Drosophila target of rapamycin dTOR. Genes Dev 14: 2712-2724.

Zhang Y, Gao X, Saucedo LJ, Ru B, Edgar BA, Pan D. 2003. Rheb is a direct target of the tuberous sclerosis tumour suppressor proteins. Nat Cell Biol 5: 578-581.

Zhang H, Liu J, Li CR, Momen B, Kohanski RA, Pick L. 2009. Deletion of Drosophila insulin-like peptides causes growth defects and metabolic abnormalities. Proc Natl Acad Sci 106: 19617-19622.

Zhou X, Zhou B, Truman JW, Riddiford LM. 2004. Overexpression of broad: A new insight into its role in the Drosophila prothoracic gland cells. J Exp Biol 207: 1151-1161. 


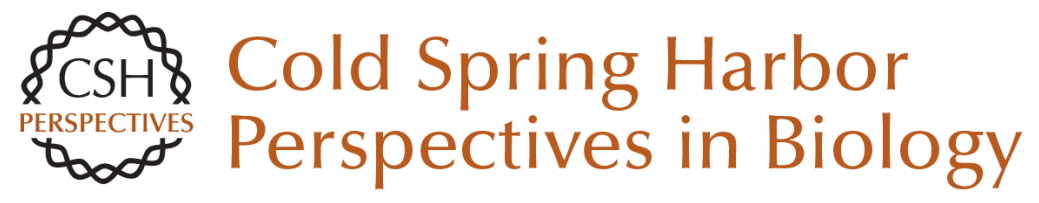

\section{The Systemic Control of Growth}

Laura Boulan, Marco Milán and Pierre Léopold

Cold Spring Harb Perspect Biol 2015; doi: 10.1101/cshperspect.a019117 originally published online August 10, 2015

\section{Subject Collection Size Control in Biology: From Organelles to Organisms}

Cell-Size Control

Amanda A. Amodeo and Jan M. Skotheim

Indeterminate Growth: Could It Represent the

Ancestral Condition?

Iswar K. Hariharan, David B. Wake and Marvalee H. Wake

The Systemic Control of Growth Laura Boulan, Marco Milán and Pierre Léopold

Genome Biology and the Evolution of Cell-Size Diversity

Rachel Lockridge Mueller

Size Scaling of Microtubule Assemblies in Early Xenopus Embryos

Timothy J. Mitchison, Keisuke Ishihara, Phuong Nguyen, et al.

The Influence of Genome and Cell Size on Brain Morphology in Amphibians Gerhard Roth and Wolfgang Walkowiak

The Opposing Actions of Target of Rapamycin and AMP-Activated Protein Kinase in Cell Growth Control

Sravanth K. Hindupur, Asier González and Michael N. Hall

Small but Mighty: Cell Size and Bacteria

Petra Anne Levin and Esther R. Angert
Mechanical Forces and Growth in Animal Tissues Loïc LeGoff and Thomas Lecuit

Biological Scaling Problems and Solutions in

Amphibians

Daniel L. Levy and Rebecca Heald

Intracellular Scaling Mechanisms Simone Reber and Nathan W. Goehring

Growing an Embryo from a Single Cell: A Hurdle in Animal Life Patrick H. O'Farrell

Organ-Size Regulation in Mammals Alfredo I. Penzo-Méndez and Ben Z. Stanger

Size Control in Plants--Lessons from Leaves and Flowers Hjördis Czesnick and Michael Lenhard

Nuclear DNA Content Varies with Cell Size across Human Cell Types

James F. Gillooly, Andrew Hein and Rachel

Damiani

Subcellular Size

Wallace F. Marshall

For additional articles in this collection, see http://cshperspectives.cshlp.org/cgi/collection/

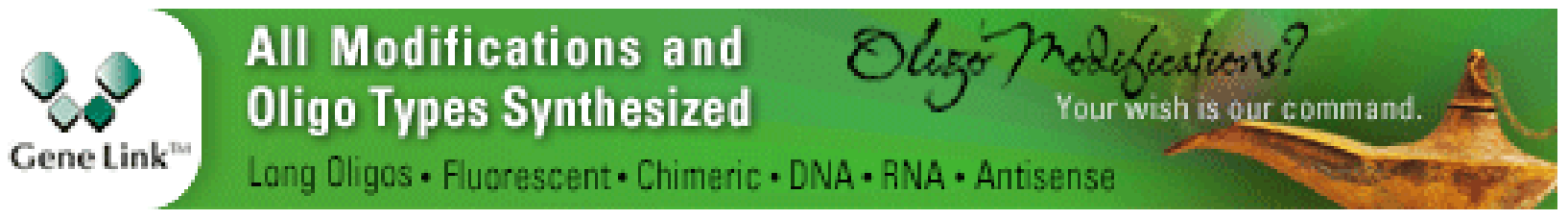

Copyright @ 2015 Cold Spring Harbor Laboratory Press; all rights reserved 